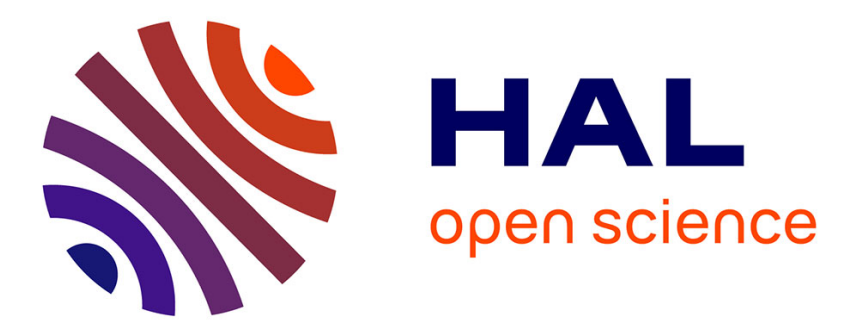

\title{
Laboratory Landquakes: Insights From Experiments Into the High-Frequency Seismic Signal Generated by Geophysical Granular Flows
}

M. Arran, A. Mangeney, J. de Rosny, M. Farin, R. Toussaint, Olivier Roche

\section{- To cite this version:}

M. Arran, A. Mangeney, J. de Rosny, M. Farin, R. Toussaint, et al.. Laboratory Landquakes: Insights From Experiments Into the High-Frequency Seismic Signal Generated by Geophysical Granular Flows. Journal of Geophysical Research: Earth Surface, 2021, 126 (5), 10.1029/2021JF006172 . hal03253991

\author{
HAL Id: hal-03253991 \\ https://hal.uca.fr/hal-03253991
}

Submitted on 18 Nov 2021

HAL is a multi-disciplinary open access archive for the deposit and dissemination of scientific research documents, whether they are published or not. The documents may come from teaching and research institutions in France or abroad, or from public or private research centers.
L'archive ouverte pluridisciplinaire HAL, est destinée au dépôt et à la diffusion de documents scientifiques de niveau recherche, publiés ou non, émanant des établissements d'enseignement et de recherche français ou étrangers, des laboratoires publics ou privés. 


\title{
Laboratory landquakes: Insights from experiments into the high-frequency seismic signal generated by geophysical granular flows
}

\author{
M. I. Arran ${ }^{1}$, A. Mangeney ${ }^{1}$, J. De Rosny ${ }^{2}$, M. Farin ${ }^{2}$, R. Toussaint ${ }^{3}$, O. \\ Roche $^{4}$ \\ ${ }^{1}$ Institut de Physique du Globe de Paris, CNRS (UMR 7154), Paris, France \\ ${ }^{2}$ Institut Langevin, ESPCI Paris, PSL Research University, CNRS, Paris, France \\ ${ }^{3}$ Institut de Physique du Globe de Strasbourg/EOST, University of Strasbourg, CNRS (UMR 7516), \\ Strasbourg, France \\ ${ }^{4}$ Université Clermont Auvergne, CNRS, IRD, OPGC, Laboratoire Magmas et Volcans, F-63000 \\ Clermont-Ferrand, France.
}

Key Points:

- We conducted novel laboratory experiments to test five existing models for the high-frequency seismic signals generated by granular flows

- The 'thin-flow' model of Farin et al. (2019) was the most accurate and makes predictions consistent with empirical observations

- The ratio between the mean and fluctuating forces exerted by a granular flow varies greatly, determined by an inertial number of the flow 


\section{Abstract}

Geophysical granular flows exert basal forces that generate seismic signals, which can be used to better monitor and model these severe natural hazards. A number of empirical relations and existing models link these signals' high-frequency components to a variety of flow properties, many of which are inaccessible by other analyses. However, the range of validity of the empirical relations remains unclear and the models lack validation, owing to the difficulty of adequately controlling and instrumenting field-scale flows. Here, we present laboratory experiments investigating the normal forces exerted on a basal plate by dense and partially dense flows of spherical glass particles. We measured the power spectra of these forces and inferred predictions for these power spectra from the models proposed by Kean et al. (2015), Lai et al. (2018), Farin et al. (2019), and Bachelet (2018), using Hertz theory to extend Farin et al. (2019)'s models to higher frequencies. The comparison of these predictions to our observations shows those of Farin et al. (2019)'s 'thin-flow' model to be the most accurate, so we examine explanations for this accuracy and discuss its implications for geophysical flows' seismic signals. We also consider the normalisation, by the mean force exerted by each flow, of the force's mean squared fluctuations, showing that this ratio varies by four orders of magnitude over our experiments, but is determined by a bulk inertial number of the flow.

\section{Plain Language Summary}

Landslides, like earthquakes, generate seismic signals: vibrations of the earth that can be detected a long way away. Analysis of the most rapid vibrations could provide information about how large a landslide is or how damaging it will be, helping emergency services respond. But full-size landslides are complex and difficult to study, so the generation of these vibrations is not yet sufficiently well understood for this information to be reliable. Therefore, in the place of full-size landslides, we studied simplified, smallscale versions in the laboratory, testing previous authors' predictions for the seismic signals they generate. We find that one set of predictions was particularly accurate and show that the corresponding predictions for full-size landslides are consistent with previous observations. This implies that a landslide's seismic signal can be used to calculate its size, its speed, and the typical size of particles within it. 


\section{Introduction}

\subsection{Background}

Landslides and other geophysical granular flows are a major natural hazard, causing on average 4000 deaths worldwide each year from 2004 to 2016 (Froude \& Petley, 2018) and an estimated billions of dollars of annual damage in the United States alone (Fleming et al., 1980; National Research Council, 1985; Schuster \& Fleming, 1986). Many areas have little or no early warning system in place (Hervás, 2003; Guzzetti et al., 2020) and a damaging event's magnitude and effects may remain unknown for hours or days after it happens (Hervás, 2003; Scholl et al., 2017), hindering the response of emergency services and preventing those in a landslide's path from making even last-minute preparations. Modelling is currently unable to remedy these knowledge gaps, with poorly constrained parameters, such as a flow's basal friction coefficient, being important in determining a landslide's runout (van Asch et al., 2007; Lucas et al., 2014; Delannay et al., 2017; Cuomo, 2020).

Better monitoring of landslide-prone areas and better modelling of flows' evolution are therefore key to the reduction of landslide hazard, and the use of seismic signals is a promising tool towards these aims. Geophysical flows exert forces on the ground over which they travel, resulting in the outwards-propagating seismic waves that Kanamori and Given (1982) first described in detail, for an event at Mount Saint Helens. These seismic waves, which we refer to as 'landquakes', can be detected by a local or regional seismic network, permitting continuous monitoring of a wide area. This monitoring suggests the possibility of early warning systems, analogous to those in use and development for earthquakes (e.g. Given et al., 2018). Furthermore, landquakes encode information about a landslide's magnitude and evolution over time, and so these seismic signals can be analysed to assess damage, to constrain model parameters, and to compare different models.

The low-frequency components of landquakes encode the accelerations of a landslide's centre of mass, and initial work successfully analysed these components both to detect landslides and to reconstruct their trajectories. Authors first linked known landslides to signals in seismic data (e.g. Galitzine (Golitsyn), 1915; Jeffreys, 1923; Peterschmitt \& de Visintini, 1964; Berrocal et al., 1978; Kanamori \& Given, 1982; Hasegawa \& Kanamori, 1987; Eissler \& Kanamori, 1987; Weichert et al., 1994) before considering 
theoretically the low-frequency signals generated by idealised landslides (e.g. Kawakatsu, 1989; Dahlen, 1993; Fukao, 1995). The theoretical framework of a single-force point source permitted the detection of landslides from seismic signals both locally (Lin et al., 2010; Chao et al., 2017) and globally (Ekström \& Stark, 2013), while inversion for that force with single-block landslide models allowed their evolution to be reconstructed or their flow parameters to be constrained (Brodsky et al., 2003; La Rocca et al., 2004; Ekström \& Stark, 2013; Allstadt, 2013; Yamada et al., 2013; Coe et al., 2016). Other authors (e.g. Favreau et al., 2010; Moretti et al., 2012, 2015, 2020; Yamada et al., 2016, 2018; Zhao et al., 2020) used a higher-order approximation towards the same aim, simulating landslides as continuous mass distributions rather than point masses and calculating the corresponding low-frequency synthetic landquakes. Comparison to observed seismic signals then permitted higher-resolution reconstruction of mass releases and flow trajectories, and the estimation of spatially varying flow parameters. However, such low-frequency seismic waves can only be detected for large landslides and, even if detected, cannot provide information on many properties relevant to landslide modelling and harm assessment, such as the size of individual particles within the flow or the vertical profiles of flow properties.

To extract more information and infer these properties, previous authors suggest using the high-frequency component of landquakes, associated with the accelerations of individual particles within the flow. The spectrogram of this high-frequency component and its envelope have distinctive shapes (Suriñach et al., 2005) which can be used to detect landslides (Hibert et al., 2014; Dammeier et al., 2016; Fuchs et al., 2018; Lee et al., 2019). Furthermore, the properties of this envelope can be related to those of the landslide: the envelope's duration to the landslide's duration and hence its loss of potential energy (Deparis et al., 2008; Hibert et al., 2011; Levy et al., 2015); the envelope's amplitude to the seismic energy emitted by the landslide and hence its volume (Norris, 1994; Hibert et al., 2011; Levy et al., 2015), its work rate against friction (Schneider et al., 2010; Levy et al., 2015), and its momentum (Hibert et al., 2015, 2017); and envelope scale and shape parameters to the landslide's geometry via multilinear regression (Dammeier et al., 2011). Without a theoretical framework for the high-frequency component of landquakes, however, both the precision and the range of validity of these relations are unclear, and it is difficult to use them to assess a landslide's damage or to better predict it. A model for landquake generation is required. 


\subsection{Existing Models}

Models of the high-frequency component of landquakes rely on the same framework: consideration of the total seismic signal as a sum of the uncorrelated signals generated by individual, random particle impacts, with the properties of the impacts determined by some mean properties of the particulate flow and with a specified Green's function mapping the force of an individual impact to the seismic signal observed at a remote station. This stochastic impact framework arises from Tsai et al. (2012)'s model of seismic noise generation from riverine sediment transport, and we discuss its validity in S2. We expect that it will be applicable to any extensive flows of stiff particles for which energetic impacts are more significant than other high-frequency sources, for signal periods smaller than the timescales over which the bulk flow varies. Examples may include avalanches, debris flows, and rockfalls involving multiple blocks.

Assuming the framework's validity, prediction of a flow's high-frequency landquake signal requires consideration of the locations $\mathbf{x} \in V$ of signal generation, and the specification of just three things at each location: 1$)$ the number $n_{I}(\mathbf{x})$ of impacts per unit volume and time; 2$)$ the force $\mathbf{F}_{I}(\mathbf{x}, t)$ applied by a single, typical impact over its duration; and 3) the Green's function $\mathbf{G}(t, \mathbf{r} ; \mathbf{x})$ for the scalar velocity response $v_{\mathbf{r}}(t)$ to that force of the seismic station detecting the signal, located at $\mathbf{r}$. Writing $\sim$ for Fourier transforms over time $\Delta t$, the landquake signal will then have power spectral density

$$
P_{v_{\mathbf{r}}}(f)=\left|\tilde{v}_{\mathbf{r}}(f)\right|^{2} / \Delta t=\int_{V} n_{I}(\mathbf{x})\left|\tilde{\mathbf{F}}_{I}(\mathbf{x}, f) \cdot \tilde{\mathbf{G}}(f, \mathbf{r} ; \mathbf{x})\right|^{2} \mathrm{~d} \mathbf{x} .
$$

\subsubsection{Direct Use of Tsai et al. (2012)}

Kean et al. (2015), Lai et al. (2018), and Farin et al. (2019) consider only impacts on exposed bedrock at the base of a flow to be significant in signal generation, and assume 1) that the rate of impacts is determined by the advection of particles, with the mean flow, into basal irregularities of the same scale; 2) that the force a particle exerts varies over timescales much shorter than the range of periods to which the seismic station is sensitive; and 3) that the relevant Green's function is that for Rayleigh-wave propagation to the far field. Under these assumptions, if a representative impacting particle has diameter $d$ and downslope speed $u$, it will have collision rate $u / d$, so that a bedrockcontacting flow area $A$ in which impacting particles have a volume fraction $\phi$ will have an approximate integrated collision rate $\int_{V} n_{I} \mathrm{~d} \mathbf{x}=\phi A u / d^{3}$. For all signal periods of 
interest, the typical force applied by an impact will be approximable as a Dirac delta function in time and hence constant in the frequency domain, equal to the impulse transferred, so that we may write $\tilde{\mathbf{F}}_{I}(f)=\Delta p \mathbf{e}_{I}$ for a unit vector $\mathbf{e}_{I}$ in the direction of the impulse. Meanwhile, the relevant frequency-space Green's function for a station at radius $r$ will have magnitude $\left|\mathbf{e}_{I} \cdot \tilde{\mathbf{G}}\right|=R(f) e^{-\alpha(f) r} / \sqrt{r}$, for functions $R$ and $\alpha$ related to Rayleighwave propagation and inelastic attenuation, respectively. Consequently, the signal's power spectral density is

$$
P_{v_{\mathbf{r}}}(f)=\frac{\phi A u \Delta p^{2}}{d^{3} r} R(f)^{2} e^{-2 \alpha(f) r} .
$$

Kean et al. (2015) suggests that $u$ scales with the measured surface velocity and $\Delta p$ with the base-normal component of the flow's local weight per unit area. The authors use an empirical, piecewise-continuous function $\alpha$, and avoid consideration of scaling constants, $\phi, R$ and $d$ by examining only the ratio of $P_{v_{\mathbf{r}}}(f)$ to that measured during a reference debris flow in the same channel, for which such parameters are assumed to be the same. The paper uses this model to estimate the depths of static sediment 'shielding' the channel centre from impacts, and these estimates correctly remain positive, but the paper performs no further evaluation of the model.

Lai et al. (2018) suggests that large, flow-depth-spanning particles dominate the signal, so that $d$ should be the 94th percentile of the particle diameter distribution and $u$ should be the depth-averaged downslope velocity $\bar{u}$ of the flow. The authors implicitly take $\phi=1$ and further assume that impacts transfer an impulse equal to that for elastic rebounds of individual near-spherical particles at vertical velocity $\bar{u}$, such that $\Delta p=\pi \rho d^{3} \bar{u} / 3$ for particle material density $\rho$. Equations for $R(f)$ and $\alpha(f)$ are taken from Tsai et al. (2012), Tsai and Atiganyanun (2014), and Gimbert and Tsai (2015), and then applied to a Californian debris flow, to invert the peak frequency of $P_{v_{\mathbf{r}}}(f)$ for $r$. However, this inversion relies on the model for signal generation only via the assumption that $\left|\tilde{\mathbf{F}}_{I}(f)\right|$ is independent of $f$ in the frequency range of interest, so this assumption is the only part of the model that the paper tests.

Farin et al. (2019) generalises the model of Lai et al. (2018) to different flow regions and regimes and to a continuous particle size distribution. The authors calculate that the impacts of particles falling from the flow front or saltating ahead of it are less significant for signal generation than those in the flow's dense snout and body. In these two regions, for 'thin' flows of depth $h$ comparable to the largest particle diameters, the pa- 
per suggests that the Lai et al. (2018) model will hold, with slight modifications: $\phi$ is explicitly stated; there are extra terms in the equation for $\Delta p$ to account for inelasticity and variation in the angle and velocity of impacts; $R$ is adjusted to account for nonvertical $\mathbf{e}_{I}$; and $d$ is represented by its appropriately weighted average over the distribution of particle diameters, which is suggested to be approximately equal to the 73rd percentile of that distribution. However, for 'thick' flows, where $h$ is much larger than the particles' diameters, the paper suggests that, in addition to the above slight modifications, the relevant advection and impact velocity is that of base-adjacent particles. Assuming no basal slip, in the sense that velocities tend to zero towards the flow's base, $u$ is then proportional to $\bar{u} d / h$ and the representative value of $d$ is equal to the 86 th percentile of the particle diameter distribution. The authors tested neither of the 'thin-flow' and 'thick-flow' models.

\subsubsection{Model of Bachelet (2018)}

In contrast to the above papers, Bachelet (2018) considers impacts between different layers of particles, throughout the depth of the flow, and supposes 1) that the local impact rate is the rate at which adjacent layers shear over each other; 2) that the force throughout an impact is described by Hertz theory with typical impact velocity equal to the standard deviation in particle velocity within each layer; and 3) that the Green's function includes exponential attenuation of the force with the impact's distance from the flow's base.

The use of Hertz theory to describe the contact force between impacting particles, detailed in S3, predicts the duration of impacts and so a frequency scale for the spectral density of the forces they exert. For a collision at relative normal velocity $u_{n}$ between two spherical particles of diameter $d$, consisting of material with density $\rho$, Young's modulus $E$, and Poisson's ratio $\nu$, Hertz theory predicts a timescale for the impact

$$
\tau=\left[\frac{\pi^{2} \rho^{2}\left(1-\nu^{2}\right)^{2}}{4 E^{2} u_{n}}\right]^{1 / 5} d
$$

With this $\tau$, the spectral density of the normal force between the particles is

$$
\left|\tilde{F}_{I}(f)\right|^{2}=\left(\frac{\pi \rho d^{3} u_{n}}{3}\right)^{2} \zeta(f \tau)
$$

for a non-dimensional function $\zeta(f \tau)$, plotted in S3, which is approximately equal to 1 for $f \tau \ll 1$, monotonically decreases to $\zeta(f \tau)=0.5$ for non-dimensional corner fre- 
quency $f_{c} \tau \approx 0.208$, and is much less than 1 for $f \tau>1$. Impacts at higher velocities $u_{n}$ apply forces with higher spectral density, over a wider frequency range.

This spectral density doesn’t appear explicitly in Bachelet (2018), which instead uses the integral of $\zeta$ over all $f \tau$ to consider the total seismic power generated by a flow. However, we can follow the thesis' reasoning to derive from equation (4) a prediction for the spectral density of a flow's high-frequency landquake signal, in the form of equation (1). First, separating a flow with representative particle size $d$ and particle volume fraction $\phi$ into layers, and writing $z_{j}$ for the vertical position of each layer and $u_{j}$ for the mean horizontal velocity within it, the thesis suggests that the rate of impacts is

$$
n_{I}(\mathbf{x})=\frac{4 \phi}{\pi d^{3}} \sum_{j}\left(u_{j}-u_{j-1}\right) \delta\left(z-z_{j}\right)
$$

for Dirac delta function $\delta$. Then, writing $T_{j}$ for the granular temperature in the $j$ th layer, equal to the variance of individual particles' velocities, the thesis takes the spectral density of the force applied by a typical impact to be given by equation (4) with impact velocity $u_{n}=\sqrt{T_{j}}$. Finally, the magnitude of the frequency-space Green's function for an impact at height $z$ is taken to be $e^{-\gamma z / 2}\left|\tilde{G}_{b}\right|$, where $\gamma$ is an attenuation constant and $\left|\tilde{G}_{b}\right|$, describing a measurement station's velocity response to vertical basal forces, is constant due to the assumption of an incoherent, diffuse seismic field with constant attenuation. Therefore, a flow of area $A$ will generate a landquake signal with power spectral density

$$
P_{v_{\mathbf{r}}}(f)=\frac{4 \phi A}{\pi d^{3}}\left|\tilde{G}_{b}\right|^{2} \sum_{j}\left(u_{j}-u_{j-1}\right) \Delta p_{j}^{2} \zeta\left(f \tau_{j}\right) e^{-\gamma z_{i}}
$$

for

$$
\Delta p_{j}=\frac{\pi \rho d^{3} \sqrt{T_{j}}}{3}, \quad \tau_{j}=\left[\frac{\pi^{2} \rho^{2}\left(1-\nu^{2}\right)^{2}}{4 E^{2} \sqrt{T_{j}}}\right]^{1 / 5} d .
$$

Bachelet (2018) shows this model to be consistent with measurements of the seismic signals generated by approximately steady and uniform laboratory-scale granular flows, but the results are not conclusive. Releasing flows of $d=2 \mathrm{~mm}$-diameter glass beads in a channel inclined at angles between $16.5^{\circ}$ and $18.1^{\circ}$, accelerometers were used to estimate the total seismic power imparted to an isolated plate by overlying flows of depths between $15 d$ and $20 d$, and this power was compared to the prediction of equation (6), with flow parameters estimated using high-speed photography through the channel's transparent sidewalls. The agreement is reasonable, but is highly dependent on the fitted parameter $\gamma$, and so the number of estimates, and their range of variation, are too 
small for conclusions to be definitive. The use of Hertz theory permits predictions for the frequency-dependence of the power spectral density, but no such predictions are compared with experimental results. Further tests are therefore required.

\subsection{Aim of This Paper}

This paper aims to test the above models of high-frequency landquake signal generation. Studying a range of granular flows and measuring the parameters used by the above models, we aim to compare observed landquake signals to the models' predictions. Because we are concerned with the generation of the signal, rather than its propagation, we consider predictions for the power spectral density $P_{F}$ of the total base-normal force exerted by the flow, which may be obtained by removing the Green's function in equation (1), so by dividing equation (2) by $R^{2} e^{-2 \alpha r} / r$ and equation (6) by $\left|\tilde{G}_{b}\right|^{2} . P_{F}$ will be proportional to the spectral density of the signal at a receiver, with its appropriately weighted integral proportional to the seismic power transmitted by the flow, but $P_{F}$, unlike these measurements, is independent of the response of the base on which the flow propagates.

However, it is difficult to use field-scale granular flows to test the models' predictions for $P_{F}$. Natural geophysical flows often occur in remote locations, infrequently and unpredictably, and so the sites of most flows are not instrumented for measurements of flow parameters. Where sites are instrumented, the destructiveness of geophysical flows restricts which parameters can be measured, excluding most used by the above models. Furthermore, geophysical flows are typically extremely unsteady and heterogeneous, so that any given landquake signal may be produced by a flow region with parameters very different from those that have been measured. Finally, the inference from a landquake signal of the forces that generated it requires inversion of the Green's function, which is typically poorly constrained at the high frequencies of interest, and to which the inversion is typically very sensitive at precisely these high frequencies.

We therefore conducted laboratory experiments to link the properties of a granular flow to the seismic signal it generates. In the laboratory, flows can be fully controlled and instrumented, allowing a wide range of parameter values to be explored and measured. Apparatus can be designed to produce steady, uniform, homogeneous flows, and the Green's function can be well constrained over a large frequency range by calibration. 
Having established the relevant physics, conclusions drawn from laboratory results can then be scaled up to describe field-scale flows.

\section{Methods}

\subsection{Experimental Apparatus}

As the simplest possible analogue of a geophysical granular flow, we studied the flow of spherical glass beads, $d=2 \mathrm{~mm}$ in diameter, in an inclined channel $2.5 \mathrm{~m}$ long and $W=0.2 \mathrm{~m}$ wide, shown in Figure 1. The beads were 1.7-2.1 mm Type $\mathrm{S}$ glass beads produced by Sigmund Lindner GmbH and provided by MINERALEX, with material density $\rho=2500 \mathrm{~kg} \mathrm{~m}^{-3}$ and Young's modulus $E=63 \mathrm{GPa}$ (Sigmund Lindner, 2018). In each experiment, $40 \mathrm{~kg}$ of beads were initially stored in a plastic reservoir of volume $0.08 \mathrm{~m}^{3}$, from which they flowed out via a rectangular opening of width $0.18 \mathrm{~m}$ and adjustable height $h_{g}$, controlled to within $0.06 \mathrm{~mm}$ by a plastic gate which was fixed in place during each experiment. A separate plastic release gate blocked this opening before each experiment and was manually lifted to start outflow. On leaving the reservoir, beads entered the separately supported channel, which had an aluminium base; transparent, $0.1 \mathrm{~m}$ high acrylic walls; and an incline $\tan \theta$, which could be adjusted by changing the heights of the braces attaching the channel to its supports. The channel's base was roughened with the same type of glass beads as constituted the flow, fixed in place with extra-strong double-sided carpet tape, with an irregular, dense pattern achieved by random pouring.

The flow of beads down the channel adjusted to these conditions over a distance of $1.92 \mathrm{~m}$, before reaching a rectangular, instrumented steel plate set into a corresponding hole in the centre of the channel's base. The plate was $X=0.18 \mathrm{~m}$ long, $Y=0.1 \mathrm{~m}$ wide, and $H=2 \mathrm{~mm}$ thick, with its surface flush with that of the aluminium base to within $0.02 \mathrm{~mm}$ and separated from it by an isolation gap of $0.04 \pm 0.01 \mathrm{~mm}$, achieved by using strips of plastic film as spacers during emplacement. The plate was supported by a force sensor and a support piece, with the three separated by washers and held together by a prestressing screw, the head of which was glued into a $0.5 \mathrm{~mm}$-deep recess in the centre of the plate's underside. The support piece, in turn, was attached to the channel's substructure using phenyl salicyclate (salol), which was added to the join when molten and solidifed to form a stiff connection, but could be melted with a heat gun for removal of the plate or adjustment of its position. Before the plate's emplacement, we 


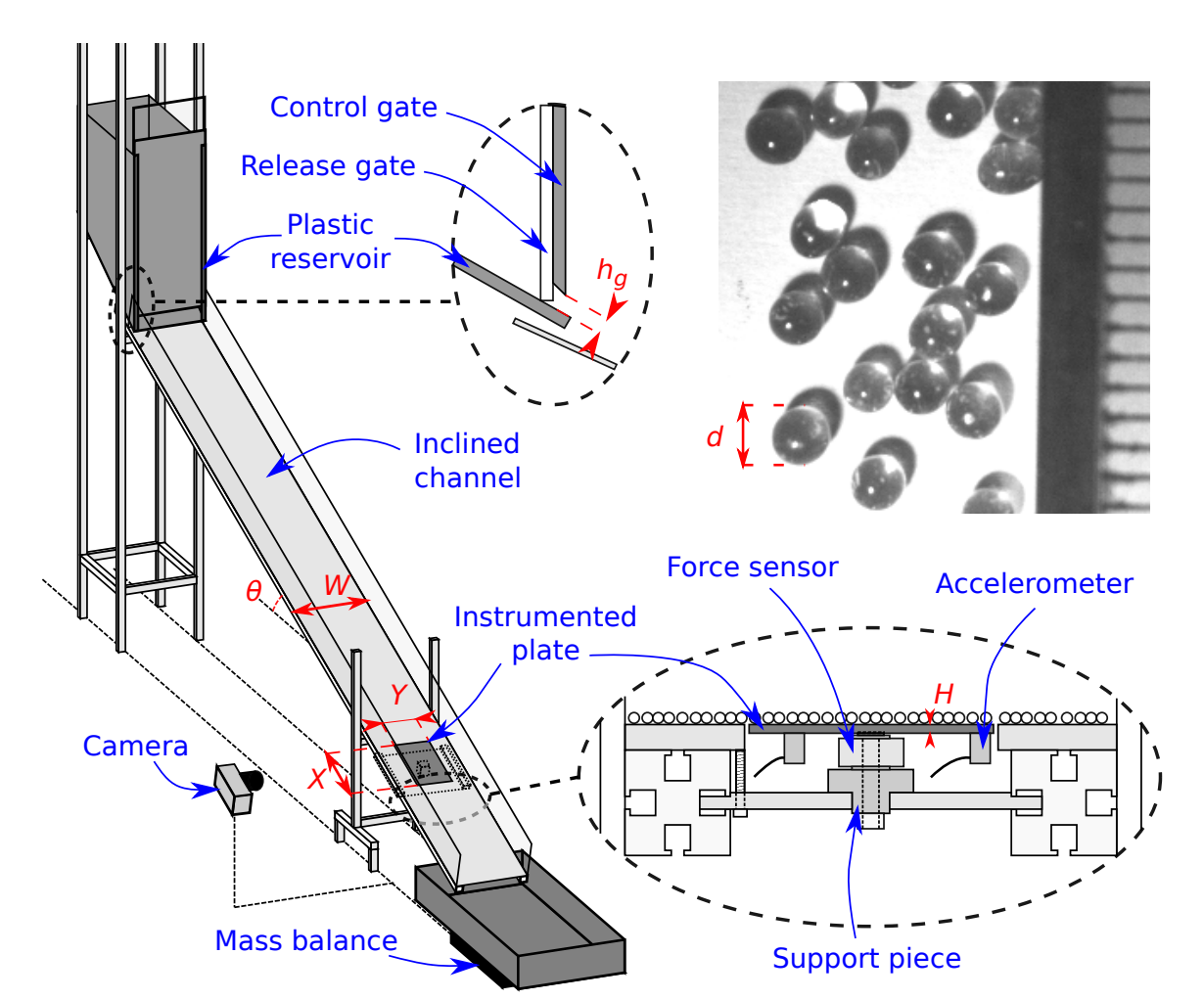
salol to form a thin, liquid layer, and we poured beads on top to form an irregular, dense pattern, before the salol solidified and fixed them in place.

After the plate, the flow of beads continued for $0.4 \mathrm{~m}$, before flowing out of the channel and into a plastic outflow tray. Plastic sheeting extended the tray's walls, to prevent energetic particles from escaping.

Figure 1. Schematic of experimental apparatus. Experiments are conducted in the channel represented, to scale, at left, with components of the apparatus labelled in blue and relevant dimensions in red. Expansions at top-centre and bottom-right represent, in cutaway views and not to scale, details of the reservoir and the instrumented plate, respectively. The glass beads used in experiments are shown at top-right, with a mm-unit scale.

used the same salol to roughen its surface with glass beads: heating the plate, we added

Four sets of devices took measurements of the flow: a mass balance beneath the outflow tray; the force sensor supporting the instrumented plate; four accelerometers attached to the plate's underside; and a high-speed camera directed through the channel's wall. The mass balance was a Dymo S50 digital shipping scale, which measured in each experiment the cumulative mass that had passed through the channel. The force sen- 
sor was a Kistler 9027C three-component force sensor and was connected to a Kistler 5073 charge amplifier, measuring the normal, downslope, and cross-slope forces exerted by the flow on the plate. The accelerometers were Brüel and Kjær type 8309 accelerometers, attached with salol to randomly selected positions on the plate's underside and connected to a Brüel and Kjær Nexus 2692-A-OS4 conditioning amplifier, to measure the normal vibrations of the plate and hence the seismic energy imparted to it by the flow. Settings of the force sensor and accelerometer amplifiers are described in S4. The camera was an Optronis CR600x2, with a Sigma 17-50 mm F2.8 EX DC lense, and was level with and focussed on the inside of the channel sidewall, directly cross-slope from the instrumented plate's centre. The camera's inclination was the same as the channel's and its field of view was $640 \times 256$ pixels, corresponding to a region $8 \mathrm{~cm}$ long and $3.2 \mathrm{~cm}$ high. The sidewall was lit using a Photonlines H5 LED light, via a white sheet of paper which acted as a reflective diffuser, and we used an exposure of $250 \mu \mathrm{s}$ and a frame rate of $2000 \mathrm{~s}^{-1}$.

To control the measurement devices, we used an Arduino Uno R3 microcontroller board, and we recorded measurements using a Pico Technology Picoscope 4824 oscilloscope connected to a Lenovo E530 laptop. Measurements from the mass balance, force sensor, and accelerometers were recorded from the time $t=0$ at which the reservoir's release gate was lifted until the outflow stopped at $t=t_{e}$, while the camera recorded footage over a duration $\Delta t_{c}$ between $2 \mathrm{~s}$ and $10 \mathrm{~s}$, after a delay time $t_{d}$ in which the flow developed into a steady state. Details are in S5.

We conducted experiments with six different channel inclinations between $22.8^{\circ}$ and $27.5^{\circ}(\tan \theta=0.42,0.44,0.46,0.48,0.50$ and 0.52$)$, with this order randomised to negate the effect of any systematic variation in atmospheric conditions or measurement sensitivity. For each inclination, we conducted three repeats with the reservoir control gate at each of four different heights $\left(h_{g}=5 \mathrm{~mm}, 10 \mathrm{~mm}, 20 \mathrm{~mm}\right.$ and $40 \mathrm{~mm}$ ), with the order of gate heights again selected at random.

At channel inclines equal to and greater than $\tan \theta=0.46\left(\theta=24.7^{\circ}\right)$, there was a gate height below which flows were in the gaseous regime of e.g. Börzsönyi and Ecke (2006) and Taberlet et al. (2007), with all glass beads in saltation and accelerating downslope. We recorded no measurements of such flows, which were energetic and far from stationary, with a large number of beads escaping across the channel's sidewalls and with 


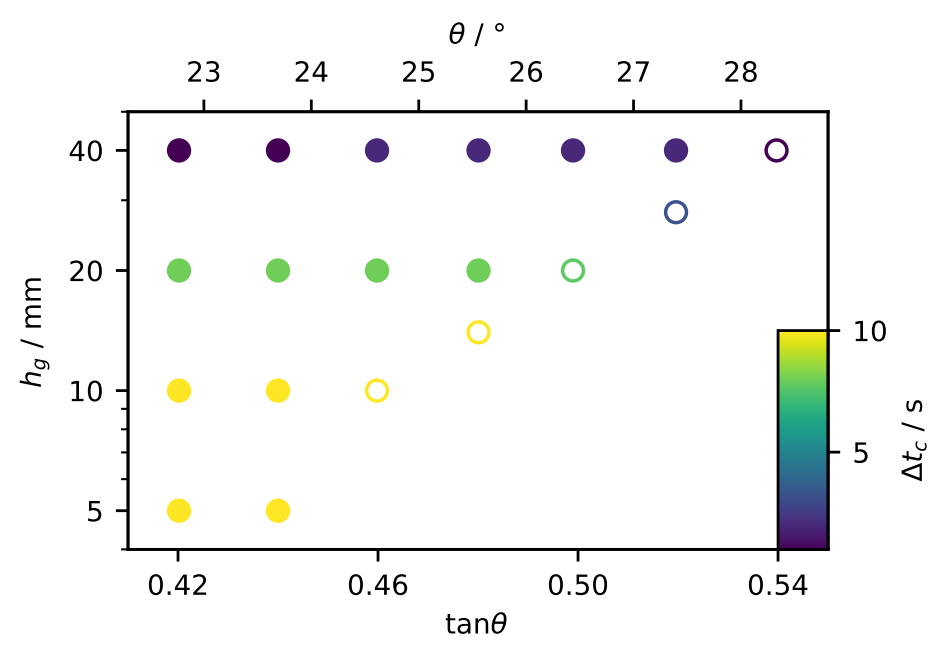

Figure 2. Channel inclines $\tan \theta$ and release gate heights $h_{g}$ used in experiments. $\circ$ indicates an experiment for which the flow was in the transitional regime, while colours indicate the duration of time $\Delta t_{c}$ recorded by the camera.

the camera's images unusable for reliable measurements. At each such incline, we instead recorded measurements at all gate heights resulting in dense flows and at one gate height resulting in a 'transitional-regime' flow, with a dense basal flow below a saltating layer. These gate heights are plotted in Figure 2, within the full parameter space investigated.

\subsection{Data Analysis}

For each experiment within the parameter space, we analysed the experimental data to calculate dynamic, seismic, and kinematic properties of the flow: the mass of particles that lay over the instrumented plate and the effective friction coefficient between the two; the mass flux of particles through the channel and their average velocity; the power spectrum of the normal force exerted on the plate by the flow; and the vertical profiles of particle volume fraction, velocity, and granular temperature at a channel wall. We recall that $W$ denotes the channel's width and $\theta$ its angle of inclination; that $X, Y$, and $H$ denote the length, width, and thickness of the plate; and that $t_{d}$ and $\Delta t_{c}$ denote the delay before and the duration of the high-speed camera's recording, respectively. These and all other variables are listed in S1 and all code used to perform these analyses is available at Arran et al. (2020). 
To infer the mass overlying the plate and its effective friction coefficient with the flow, we used the data from the force sensor. Averaging over successive $0.5 \mathrm{~ms}$ intervals, the net downslope force $F_{x}(t)$ and plate-normal, downwards force $F_{z}(t)$ applied to the plate by the flow were calculated from the voltage output of the force sensor's charge amplifier, as described in S6. Then, assuming no net plate-normal acceleration of the flow overlying the plate, over the period of steady flow recorded by the camera, we calculated the average mass per unit area overlying the plate as

$$
\sigma=\frac{\left\langle F_{z}\right\rangle_{\Delta t_{c}}}{X Y g \cos \theta}
$$

where $\langle\cdot\rangle_{\Delta t_{c}}$ represents the arithmetic mean over $t_{d}<t<t_{d}+\Delta t_{c}$ and $g$ represents gravitational acceleration. Similarly, we followed Hungr and Morgenstern (1984) and Roche et al. (2020) in calculating the effective friction coefficient as

$$
\mu=\frac{\left\langle F_{x}\right\rangle_{\Delta t_{c}}}{\left\langle F_{z}\right\rangle_{\Delta t_{c}}}
$$

with this calculation validated in S6, section S6.3.

To calculate the mass flux through the channel, we examined the data recorded by the mass balance. Having the cumulative mass $M(t)$ that had flowed through the channel after time $t$, we calculated the average flux per unit channel width, over the period of steady flow recorded by the camera, as

$$
q=\frac{M\left(t_{d}+\Delta t_{c}\right)-M\left(t_{d}\right)}{\Delta t_{c} W} .
$$

Assuming this average mass flux to be equal to that across the plate, and having calculated the mass overlying the plate, we could then calculate the mean depth-averaged flow velocity across the plate,

$$
\bar{u}=q / \sigma
$$

To extract the power spectral density of the flow's basal force, we processed data from the accelerometers using Kirchhoff-Love plate theory and assuming perfect isolation of the plate from the channel and linear attenuation within the plate. On the basis of the steel's technical documentation (John Steel, 2019; Steel SS, 2019), we took its density to be $\rho_{p}=7800 \mathrm{~kg} \mathrm{~m}^{-3}$, its Young's modulus to be $E_{p}=200 \mathrm{GPa}$, and its Poisson's ratio to be $\nu_{p}=0.29$. Then, its bending stiffness was $D=E_{p} H^{3} / 12\left(1-\nu_{p}^{2}\right)$ and the mean bandgap between its resonant frequencies was $\Delta_{f}=2 \sqrt{D} / X Y \sqrt{\rho_{p} H} \approx 400 \mathrm{~Hz}$. 
Assuming that the spectral density of an impact's force varied little over this frequency scale, this spectral density was estimated using $D$, the proportion of the plate's energy $\mathcal{P}$ in its steel structure's vertical displacements, the quality factor $Q$ describing the attenuation of energy in the plate, and the accelerations $a_{j}(t)$ measured by the four accelerometers, as

$$
P_{F}(f)=\frac{|\tilde{F}(f)|^{2}}{\Delta t} \approx \frac{\left(\rho_{p} H\right)^{3 / 2} X Y \sqrt{D}}{\pi \mathcal{P} Q f \Delta t}\left\langle\sum_{j=1}^{4}\left|\tilde{a}_{j}(f)\right|^{2}\right\rangle_{\Delta f},
$$

where Fourier transforms are over a time interval $\Delta t=0.2 \mathrm{~s}$, and $\langle\cdot\rangle_{\Delta f}$ represents a moving average over frequency, with window width $\Delta f=2 \mathrm{kHz}$. We describe in S7, section S7.1 the derivation of this relation and the calculation of $\left|\tilde{a_{j}}\right|^{2}$ from the voltage output of the accelerometers' conditioning amplifer; in section S7.2 the calibration we performed to measure the plate parameters $\mathcal{P}=0.25$ and $Q=99$ and to extend the flat frequency range of the accelerometers to $120 \mathrm{kHz}$; and in section $\mathrm{S} 7.3$ the validation of this work.

Finally, to extract profiles of kinematic properties at the channel wall, we analysed the images taken by the high-speed camera, using particle tracking velocimetry and Gaussian coarse-graining. Analysing each frame in turn, we detected the positions $\left(x_{j}, z_{j}\right)$ of particles at the channel walls and, tracking particles between consecutive frames, calculated their mean velocities over each $0.5 \mathrm{~ms}$ interval. Calculating the smoothed velocities $\mathbf{u}_{j}$ over five frames, or $2.5 \mathrm{~ms}$, we estimated the downslope-averaged and time-averaged base-normal profiles at the channel's wall of relative volume fraction $\phi_{w}(z)$, mean velocity $\mathbf{u}_{w}(z)$, and granular temperature $T_{w}(z)$ as

$$
\begin{aligned}
\phi_{w}(z) & =\left\langle\sum_{j} C\left(z_{j} ; z\right) \pi d^{2} / 4\right\rangle_{\Delta t_{c}}, \\
\mathbf{u}_{w}(z) & =\left\langle\sum_{j} C\left(z_{j} ; z\right) \pi d^{2} \mathbf{u}_{j} / 4\right\rangle_{\Delta t_{c}} / \phi_{w}(z), \\
T_{w}(z) & =\left\langle\sum_{j} C\left(z_{j} ; z\right) \pi d^{2}\left\|\mathbf{u}_{j}-\mathbf{u}_{w}(z)\right\|^{2} / 4\right\rangle_{\Delta t_{c}} / \phi_{w}(z),
\end{aligned}
$$

where averages are over all frames recorded by the camera, sums are over all particles tracked in each frame, and the coarse-graining function $C$ is localised around $z$, with integral over the total spatial domain equal to 1. This process is described in detail in S8.

While the irregularity of the flow's base and surface complicate the definition of the flow thickness $h$, we take the base-normal co-ordinate $z$ to be zero at the top of the 
base's fixed beads, and extract $h$ as the value of $z$ at which $\phi_{w}(z)$ drops below half its maximum value,

$$
h=\min \left\{z>\operatorname{argmax} \phi_{w} \mid \phi_{w}(z)<\max \left(\phi_{w}\right) / 2\right\} .
$$

For a flow with constant particle volume fraction below a level surface, this exactly corresponds to the intuitive flow depth. Whilst other reasonable definitions lead to different specific results, they do not alter our conclusions.

\subsection{Model Predictions}

For each of the models described in section 1.2, for a granular flow's seismic signal, we inferred predictions for the experimental seismic signal. Specifically, we expressed a prediction $\hat{P}_{F}$ for the power spectrum of the base-normal force applied by the flow to the instrumented plate, as a function of the flow properties specified in section 2.2: the mean depth-averaged flow velocity $\bar{u}$, the mass overburden per unit area $\sigma$, the flow depth $h$, and the channel-wall profiles $u_{w}(z)$ and $T_{w}(z)$ of downslope velocity and granular temperature. Since previous authors attempted to predict slightly different seismic properties and used slightly different flow properties, no directly applicable expressions are in the articles introducing the models (Kean et al., 2015; Lai et al., 2018; Farin et al., 2019; Bachelet, 2018). We therefore worked from equations (2) and (6); used the models' methods of estimating those equations' variables, as described in sections 1.2.1 and 1.2.2; and removed Green's functions as described in section 1.3, to predict the basal force's power spectrum rather than the power spectrum of a seismic station's response. Recalling that $g \cos \theta$ denotes base-normal gravitational acceleration, $d$ the particles' mean diameter, and $\rho$ their density, and approximating the flow area generating the measured signal by the instrumented plate's area $A=X Y$ and the flow's mean volume fraction by $\phi=$ $\sigma / \rho h$, these predictions could then be compared to the measured power spectra $P_{F}$.

The model introduced by Kean et al. (2015) predicts the seismic signal generated by a granular flow covering a certain area, using its surface velocity and the base-normal component of its weight per unit area. If the near-base velocity of the flow scales with its surface velocity, as Kean et al. (2015) suggests, then both will scale with the depthaveraged velocity $\bar{u}$, so to calculate predictions we estimated the velocity $u$ of equation (2) with $\bar{u}$ and the impulse $\Delta p$ with $\sigma g \cos \theta$, the measured base-normal component of the flow's weight per unit area. We may therefore write the model's prediction for $P_{F}$, 
for signal periods $1 / f$ well above the duration of a typical impact, as

$$
\hat{P}_{F}^{0}=K A \bar{u}(\sigma g \cos \theta)^{2} / d^{3} .
$$

$K$ is a free parameter supposed equal to the product of a constant volume fraction; a constant of proportionality between $\bar{u}$ and the near-base flow velocity; and a squared constant of proportionality between the mean basal pressure and the typical impulse transferred by a basal impact. No indication is given as to its value, so it must be found by fitting.

In contrast, the model introduced by Lai et al. (2018) requires no free parameter. Noting that the experimental particles have a narrow diameter distribution, with 94th percentile approximately equal to its mean $d$, and using the appropriate substitutions for $u$ and $\Delta p$ in equation (2), the model's prediction for $P_{F}$ is the constant

$$
\hat{P}_{F}^{0}=\pi^{2} \rho^{2} A d^{3} \bar{u}^{3} / 9
$$

with the implicit assumption that the volume fraction is equal to 1. Again, this prediction is expected to be valid only for signal periods $1 / f$ well above the duration of a typical impact.

The two models described by Farin et al. (2019) are developments of this model, with that article's equation (16) developing the definition of the impulse denoted $\Delta p$ in our equation (2). Within the same frequency range as in prior paragraphs, the associated predictions for $P_{F}(f)$ are the constants

$$
\hat{P}_{F}^{0}=\pi^{2} \rho^{2} \phi A d^{3}(1+e)^{2} \xi(v) u_{b}^{3} / 36
$$

where $e$ is a constant coefficient of restitution; $\xi(v) \approx 0.053\left(1+5.6 v^{2}\right)$ is a non-dimensional function accounting for variation in impacts' geometry; and $v$ and $u_{b}$ define the velocities of base-impacting particles $u_{b}\left(\mathbf{e}_{x}+v \mathbf{e}_{v}\right)$, for randomly directed unit vector $\mathbf{e}_{v}$.

In the 'thin-flow' model, $u_{b}=\bar{u}$, whereas in the 'thick-flow' model $u_{b}=\chi \bar{u} d / h$ for velocity profile shape factor $\chi$, assumed constant and between 1 and 1.5. The modelspecific parameters are $e, v$ and $\chi$, which all contribute to the prefactor and so cannot be individually determined via fitting. We therefore take $e=0.9$, consistent with the rebound heights of particles dropped onto the instrumented plate; take $\chi=1.25$, consistent with the velocity profiles measured at the channel's wall; and fit the free param- 
eter $v$, corresponding to the normalised standard deviation of base-impacting particles' velocities. Farin et al. (2019)'s derivation of $\xi$ makes unphysical assumptions (e.g. that impacting particles' velocities differ from $u_{b} \mathbf{e}_{x}$ by an exactly constant magnitude $v u_{b}$ and that, for each impact velocity, all possible impact locations are equally likely), so the bestfit value of $v$ for an otherwise-accurate model will not exactly equal the true normalised standard deviation, but a model cannot be said to be accurate unless this best-fit value is a physically reasonable approximation. Specifically, the energy associated with velocity fluctuations is drawn from the mean flow and dissipated rapidly, so that we expect the typical magnitude of velocity fluctuations to be less than the mean velocity, and hence a condition for model accuracy is that $v<1$.

To further assess the assumptions of the 'thick-flow' and 'thin-flow' models, we extended each model to higher frequencies. Farin et al. (2019) assumes binary, elastic, normal interactions during impacts, with impact velocities such that particle deformation in our experiments will be quasistatic and the Hertz theory described in S3 will apply. Applying this theory to the impact velocities and geometry assumed by Farin et al. (2019), we therefore compute predictions for $P_{F}$ over a larger frequency range than that considered by the original article, as

$$
\hat{P}_{F}(f)=\frac{\int_{S^{2}} \mathrm{~d}^{2} \mathbf{e}_{v} \int_{S_{\pi / 6}^{2}} \mathrm{~d}^{2} \mathbf{e}_{n}\left(u_{n} \mathbf{e}_{n} \cdot \mathbf{e}_{z}\right)^{2} \zeta(f \tau) \mathcal{H}\left(u_{n}\right)}{\int_{S^{2}} \mathrm{~d}^{2} \mathbf{e}_{v} \int_{S_{\pi / 6}^{2}} \mathrm{~d}^{2} \mathbf{e}_{n}\left(u_{n} \mathbf{e}_{n} \cdot \mathbf{e}_{z}\right)^{2} \mathcal{H}\left(u_{n}\right)} \hat{P}_{F}^{0}
$$

for unit sphere $S^{2}$; unit spherical cap $S_{\pi / 6}^{2}$ with maximum polar angle $\pi / 6$; normal impact velocity $u_{n}=u_{b}\left(\mathbf{e}_{x}+v \mathbf{e}_{v}\right) \cdot \mathbf{e}_{n}$; impact timescale $\tau\left(u_{n}\right)$ as defined by equation (3); non-dimensional function $\zeta$ as introduced in equation (4); and Heaviside step function $\mathcal{H}$.

Finally, the model described in Bachelet (2018) already predicts $P_{F}$ over a large frequency range. Substituting equations (7) into (6) and moving from the well-defined particle layers considered in the thesis to the continuous profiles measured in our experiments, the predicted power spectral density of the basal force is

$$
\hat{P}_{F}(f)=\frac{4 \pi}{9} \phi A \rho^{2} d^{3} \int_{0}^{h} u_{w}^{\prime}(z) T_{w}(z) \zeta\left(f \tau_{w}(z)\right) e^{-\gamma z} \mathrm{~d} z
$$

where $u_{w}^{\prime}$ is the derivative of $u_{w} ; \zeta$ is the non-dimensional function in equation (4); impact timescale $\tau_{w}(z)$ is defined with respect to $T_{w}(z)$ as $\tau_{j}$ is to $T_{j}$ in equation (7); and constant $\gamma$ is a free parameter, to be determined by fitting. 
We compare these predictions to the measured power spectra $P_{F}$ in section 3.3 , but first we define the time period and the frequency-space properties used for the comparison, by considering the evolution of the flow (section 3.1) and the form of the power spectrum of the basal force (section 3.2).

\section{Results}

\subsection{Evolution of the Flow}

In each experiment, the flow passing a given point evolved through four distinct stages: I) precursory saltation of particles released at the start of the experiment; II) arrival of the dense flow's front; III) steady flow; and IV) decay of the flow. These corresponded to different signals measured at the instrumented plate, as illustrated for two different experiments in Figure 3.

As Figure 3 illustrates, saltating particles in stage I contributed little to the outflow mass $M$ and to the net downslope and normal forces $F_{x}$ and $F_{z}$, with an implied number density of around one particle per $\mathrm{cm}^{2}$ of plate surface, but such particles applied basal forces with significant spectral density $P_{F}$ across a wide frequency range. Similarly, the dense front's arrival in stage II had a short duration, but was associated with an intense, broad power spectrum of basal force, as high-velocity, surficial particles reached the front and impacted the plate. In general, as in Figure 3a, the power spectrum at high frequencies then dropped during stages III and IV, indicating that impact velocities in the dense flow's bulk were lower than those of high-velocity saltating particles. For 'transitionalregime' flows, however, $P_{F}$ remained the same during stages II and III, as in Figure 3b, reflecting the continued saltation within each flow that defines this regime.

Such variation of signal properties between different experiments is summarised in Table 1. With increasing channel incline $\tan \theta$ and release gate height $h_{g}$, the duration of stage I decreased rapidly and that of stage II decreased slightly, as the speed of the dense flow front increased to the speed of saltating particles. Since the same changes greatly increased the high-frequency spectral density $P_{F}$ of the plate-normal force during stage III, which had duration determined by the reservoir's capacity and decreasing with $h_{g}$, the contribution of stage I to the total generation of seismic energy decreased from around $70 \%$ to less than $0.1 \%$, while the contribution of stage II remained between around $10 \%$ and $20 \%$, and the contributions of stages III and IV increased. In contrast to this pat- 


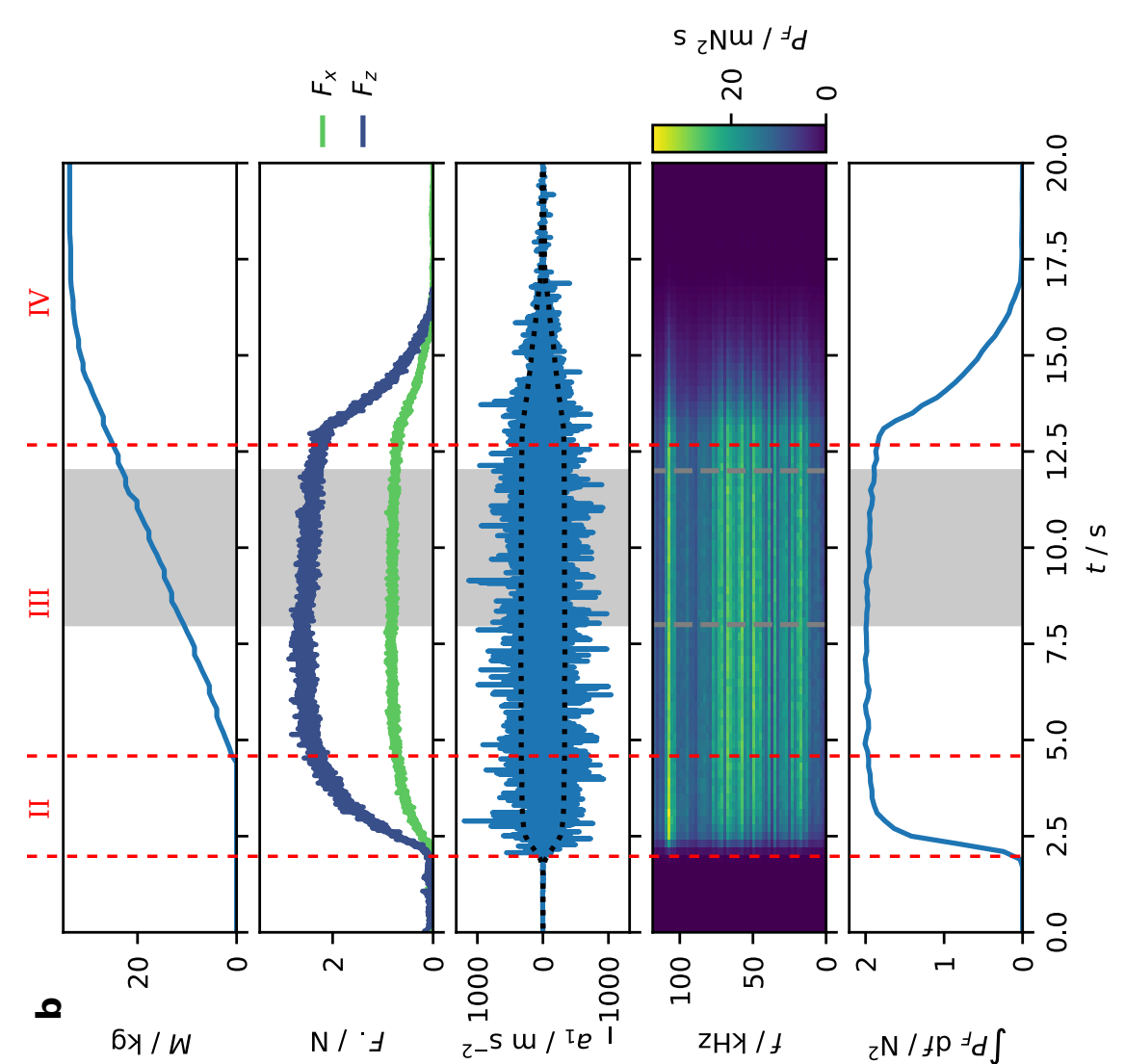

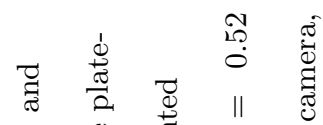

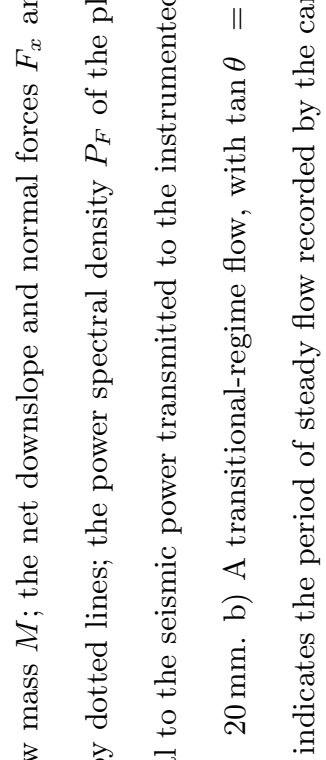

密

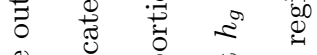

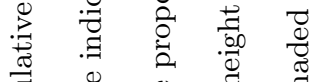

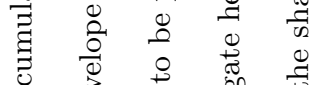

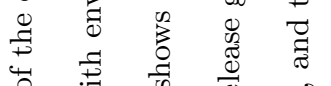

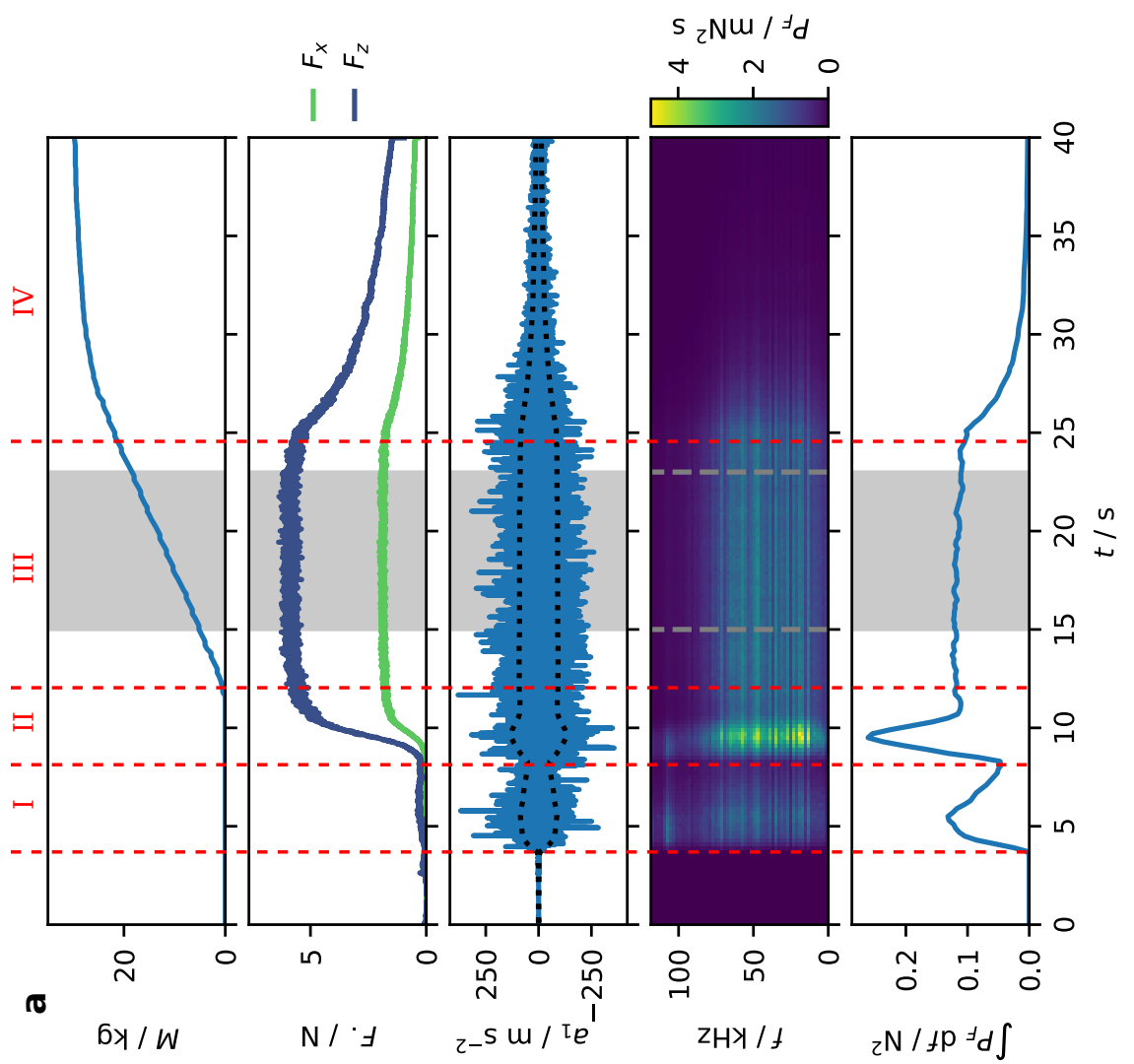

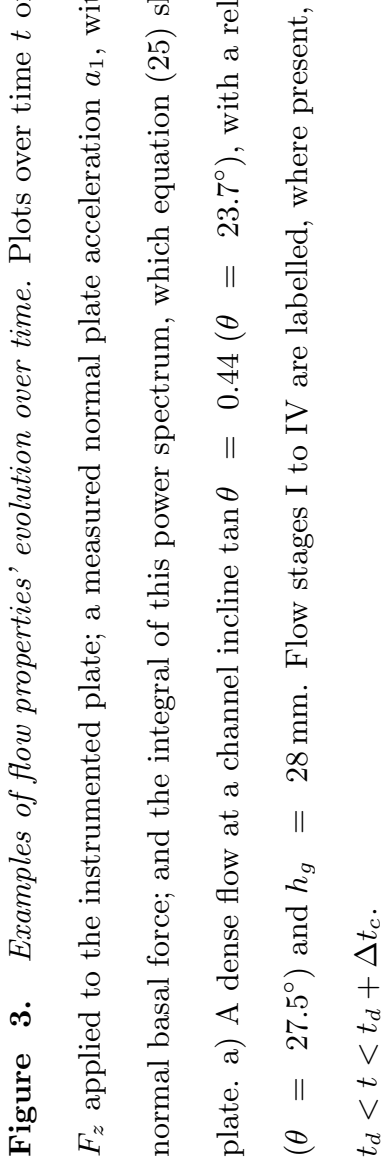


Table 1. Properties of the flow's stages of evolution. $F_{z}$ and $P_{F}$ are as defined in section 2.2, while $f_{c}$ is the frequency at which $P_{F}$ drops to half its mean value pre-maximum. Arrows $(\rightarrow)$ indicate ranges over time in an experiment, while hyphens $(-)$ represent the ranges over different experiments. • indicates the value for dense flows and $\circ$ for transitional-regime flows, wherever they differed significantly.

\begin{tabular}{lllll}
\hline Flow stage & $\mathrm{I}$ & $\mathrm{II}$ & $\mathrm{III}$ & $\mathrm{IV}$ \\
\hline Durations $/ \mathrm{s}$ & $0-40$ & $2-8$ & $4-150$ & $5-20$ \\
$F_{z} / \mathrm{N}$ & $O(0.1)$ & $0 \rightarrow(1-10)$ & $1-10$ & $(1-10) \rightarrow O(1)$ \\
& & & $\bullet 70-110$ & $\bullet 70-110$ \\
$f_{c} / \mathrm{kHz}$ & $>100$ & $>90$ & $\circ>100$ & $\circ>100$ \\
& & & $0.003-3$ & $(0.003-3) \rightarrow 0$ \\
\hline$P_{F} \mathrm{~d} f / \mathrm{N}^{2}$ & $O(0.1)$ & $0.02-2$ & & \\
\hline
\end{tabular}

tern of variation, the net normal force $F_{z}$ increased with $h_{g}$ but, for each $h_{g}$, decreased with $\tan \theta$, as the same flux of particles was maintained by a thinner flow, travelling faster. These opposing trends indicate the independence of $F_{z}$ and $P_{F}(f)$ for $f \neq 0$, with the former the mean force applied by the flow, and the latter associated with the force's fluctuations about this mean.

In this article, we restrict our attention to stage III of the flow's evolution, in which the flow's steadiness ensured that all measurements were of the same flow state. Specifically, between different $\Delta t=0.2 \mathrm{~s}$ time intervals within the duration $\Delta t_{c}$ of steady flow recorded by the camera, the per-second rate of change of outflow mass $M$ had a standard deviation of around $10 \%$ of its mean value, while the standard deviations of $F_{x}$ and $F_{z}$ were around $1 \%$ and that for $\int P_{F} \mathrm{~d} f$ around $5 \%$. Similarly, we examined the profiles of kinematic properties at the channel wall, averaged in turn over each decile of time $t_{d}+n \Delta t_{c} / 10<t<t_{d}+(n+1) \Delta t_{c} / 10$ within the period recorded by the camera. Within the flow, kinematic properties at the channel wall were steady over time, in the sense that the values of $\phi_{w}(z), \mathbf{u}_{w}(z)$ and $T_{w}(z)$ varied by at most a few percent over time, for each $z$ satisfying $\phi_{w}(z)>\max _{z} \phi_{w}(z) / 2$. 


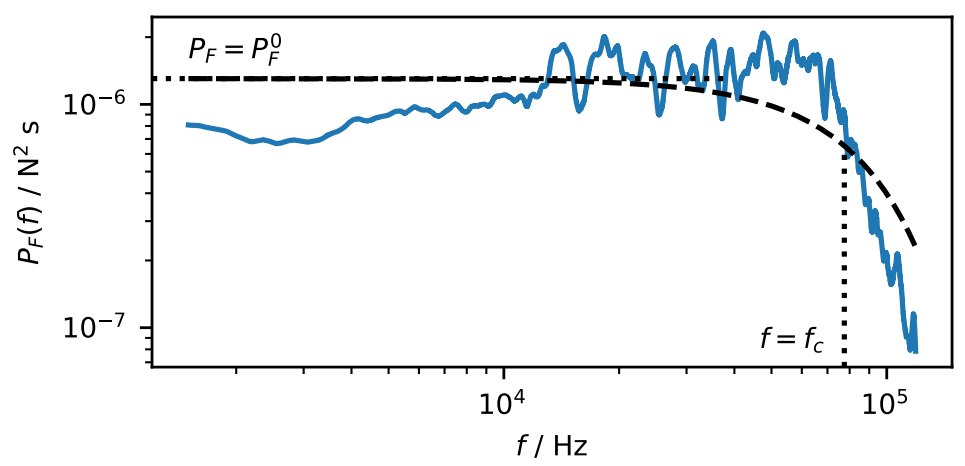

Figure 4. Example of the plate-normal force's power spectral density during steady flow.

The power spectrum corresponds to the same experiment as Figure 3a, with channel incline $\tan \theta=0.44\left(\theta=23.7^{\circ}\right)$ and release gate height $h_{g}=20 \mathrm{~mm}$. The dotted lines indicate the corner frequency $f_{c}=77.5 \mathrm{kHz}$ and the low-frequency amplitude $P_{F}^{0}=1.29 \mathrm{mN}^{2} \mathrm{~s}$. The dashed line indicates the Hertzian power spectrum fit to these values, corresponding to 4000 Hertzian impacts per second of the experimental particles on the plate's surface, each at normal velocity $0.9 \mathrm{~m} \mathrm{~s}^{-1}$.

\subsection{Power Spectrum of the Basal Force}

Averaging over this period of steady flow, by taking $\Delta t=\Delta t_{c}$ in equation (12), we calculated the power spectrum of the base-normal force applied by the flow to the plate and find it to be consistent with impacts of short duration. As in the example shown in Figure 4, the power spectral density $P_{F}(f)$ is approximately constant over a large frequency range and displays the same decay beyond a given corner frequency as Hertz theory predicts for a single impact. The fluctuations around this trend with varying frequency $f$ are consistent between different experiments, as may be seen in Figure 3, and are systematic errors arising from variation in the density of the plate's resonant modes in frequency space, as discussed in S7.

We described the power spectrum by two quantities: its low-frequency amplitude $P_{F}^{0}$ and its corner frequency $f_{c}$. We calculated $f_{c}$ as the frequency at which $P_{F}(f)$ drops to half its mean pre-maximum value, so that for a Hertzian impact it would be equal to approximately $0.208 / \tau$, for the timescale $\tau$ defined in section 1.2 .2 and S3. Any such comparison between $f_{c}$ and a theoretical corner frequency is unlikely to be exact, due to systematic errors in our measured power spectra $P_{F}$, and this is reflected by the mismatch 
Table 2. Summary of model testing. For each of the existing models described in section 1.2, we list those of the flow measurements defined in section 2.2 that are required to predict the flow's high-frequency seismic signal. We further record the equation for predictions $\hat{P}_{F}^{0}$; the free parameter value for which such predictions best fit measurements; and the geometric standard error $\epsilon=\exp \left[\sqrt{\frac{1}{N} \sum \ln \left(P_{F}^{0} / \hat{P}_{F}^{0}\right)^{2}}\right]$ of these predictions.

\begin{tabular}{lllll}
\hline Model & Inputs & Equation & Best-fit parameter & $\epsilon$ \\
\hline Kean et al. (2015) & $\bar{u}, \sigma, \theta$ & $(17)$ & $K=4.0 \times 10^{-16} \mathrm{~m}^{4} \mathrm{~s}^{2}$ & 4.2 \\
Lai et al. (2018) & $\bar{u}$ & $(18)$ & $\mathrm{n} / \mathrm{a}$ & 18.5 \\
Farin et al. (2019) & $\bar{u}, \sigma, h$ & $(19)$ & & 3.2 \\
'thick-flow' & & & $v=9.8$ & 2.1 \\
'thin-flow' & & & \\
Bachelet (2018) & $\sigma, h, u_{w}, T_{w}$ & $(21)$ & $\gamma=0.51$ & 3.9 \\
\hline
\end{tabular}

between theoretical and experimental results in Figure 4. However, our measurements of $f_{c}$ were sufficiently robust that we calculated $P_{F}^{0}$ as the mean value of $P_{F}$ over all frequencies less than $f_{c} / 2$. We could then compare these experimentally measured values with the model predictions, computed as described in section 2.3 .

\subsection{Tests of Existing Models for Flows' Seismic Signals}

To assess the model predictions described in section 2.3, we compared their predictions $\hat{P}_{F}^{0}$ for the low-frequency value of the basal force's power spectrum to the measured values $P_{F}^{0}$. Where possible, we also inferred a prediction $\hat{f}_{c}$ for the corner frequency of the basal force's power spectrum, as the frequency at which $\hat{P}_{F}(f)$ dropped to half its maximum value, and we compared this prediction with the measured value $f_{c}$. Where a model had a free parameter, we used the parameter value that minimised the sum over all experiments of $\ln \left(P_{F}^{0} / \hat{P}_{F}^{0}\right)^{2}$, which was equivalent to minimising the typical logarithmic error or maximising the model likelihood under the assumption that measurements were log-normally distributed about their predicted values (see S9). Table 2 lists these best-fit parameter values and Figure 5 shows the results of the comparisons. 

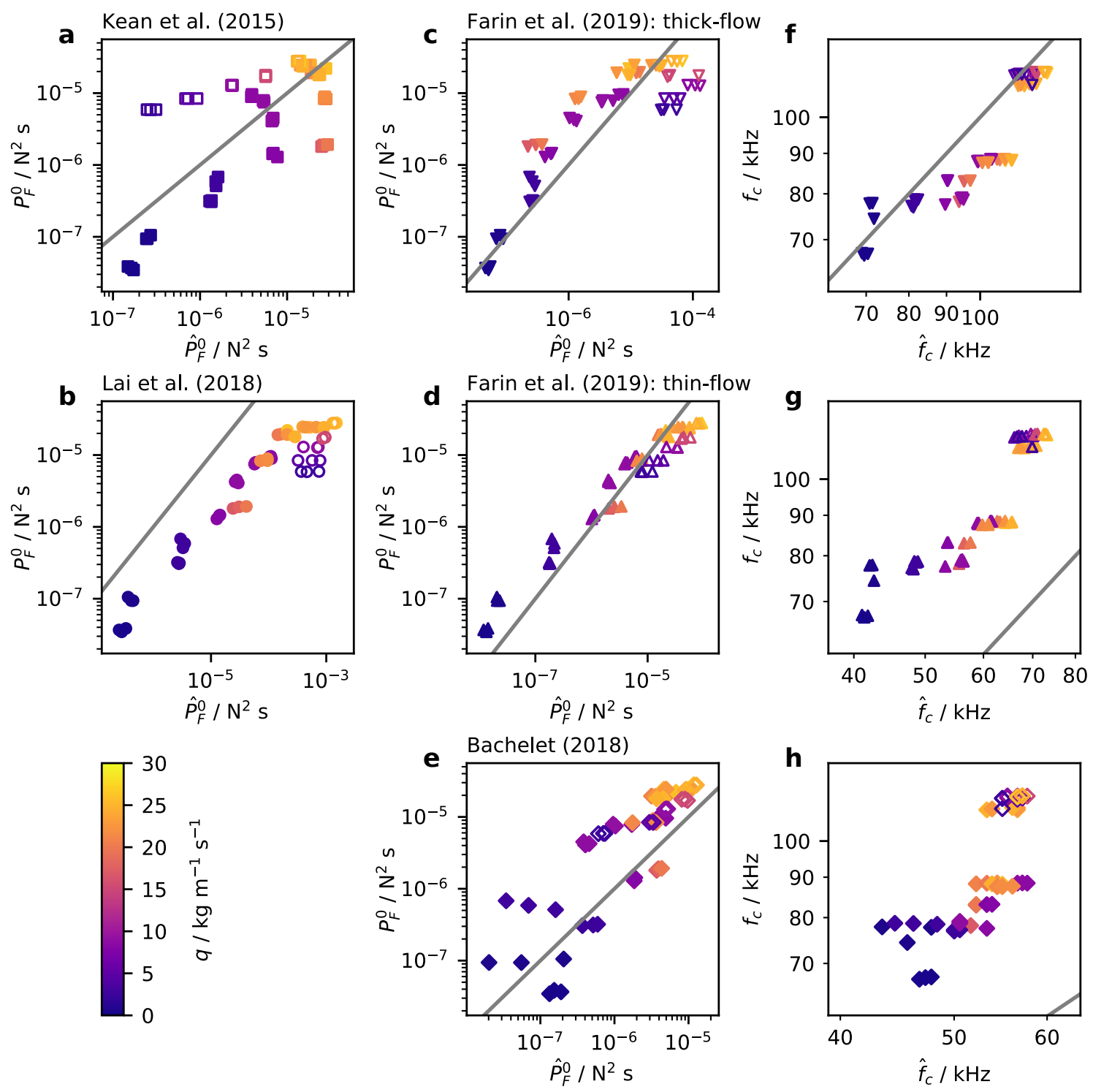

Figure 5. Comparison between models' predictions (x-axes) and experimental measurements (y-axes) for the basal force's power spectrum. Plots $\mathrm{f}, \mathrm{g}$, and h represent predictions for the corner frequency of the basal force's power spectrum, while all others represent predictions for the power spectral density's value at frequencies well below this corner frequency. In all plots, the grey line represents perfect agreement between predictions and measurements, colours indicate each experiment's mass flux $q$ per unit channel width, and unfilled symbols represent experiments for which the flow was in the transitional regime. 
The model introduced by Kean et al. (2015) predicts $P_{F}^{0}$ poorly, due largely to its incorrect assumption of proportionality between the pressure fluctuations relevant to $P_{F}^{0}$ and the mean pressure $\sigma g \cos \theta$ used as input. To best fit the measurements, the free parameter $K$ had to take a value of $4.0 \times 10^{-16} \mathrm{~m}^{4} \mathrm{~s}^{2}$, with a magnitude unforeseeable from the model's assumptions, and even then predictions often differed from measurements by an order of magnitude (Figure 5a). Notably, the model's predictions $\hat{P}_{F}^{0}$ decrease for flows at higher channel inclinations or in the transitional regime, for which the mean pressure is lower, whereas such flows' higher impact energies in fact resulted in higher pressure fluctuations and so larger measured values $P_{F}^{0}$.

In contrast, the model introduced by Lai et al. (2018) accurately predicted variation in $P_{F}^{0}$ between experiments, with predictions for dense flows consistently 3 to 10 times larger than the measured values (Figure 5b). For transitional-regime flows, the predictions' errors are larger, due to the model's implicit assumption that the volume fraction is equal to one.

Surprisingly, of the two models described by Farin et al. (2019), the model derived for flows thicker than the largest particles is less accurate than that derived for thin flows, with the former requiring an unphysical best-fit value for the ratio $v$ between the magnitudes of velocity fluctuations and of the mean velocity. For the 'thick-flow' model, we required $v=9.8$ for predictions $\hat{P}_{F}^{0}$ to be as large as measurements $P_{F}^{0}$ and, in that case, the predictions were too large for the transitional-regime flows (Figure 5c). For the "thinflow' model, meanwhile, the best-fit value was $v=0.51$, which is physically reasonable and provides an excellent fit of $\hat{P}_{F}^{0}$ to $P_{F}^{0}$ over all experiments (Figure $5 \mathrm{~d}$ ).

This difference between the 'thick-flow' and 'thin-flow' models' best-fit values of $v$ is reflected in the predictions $\hat{f}_{c}$ they implied for the corner frequency of the basal force's power spectrum, calculated according to our extensions of these models using equation (20). The higher $v$ required for the 'thick-flow' model results in higher predictions $\hat{f}_{c}$, matching the measured values $f_{c}$ (Figure $5 \mathrm{f}$ ), whereas for the 'thin-flow' model predictions are consistently approximately $30 \%$ smaller than the measured values (Figure $5 \mathrm{~g}$ ). Predicted corner frequencies $\hat{f}_{c}$ are as large as measurements $f_{c}$ only for typical impact velocities six times larger than the flows' mean velocities, suggesting that our measurements $f_{c}$ were slight, but systematic, overestimates. 
Finally, the predictions of the model described in Bachelet (2018) followed the correct trend but had a wide dispersion (Figure 5e). The free parameter $\gamma$, representing signal attenuation within the flow, had best-fit value 0 , indicating that the unattenuated contributions of all synthetic impacts are necessary for $\hat{P}_{F}^{0}$ to be large enough to compare to $P_{F}^{0}$. Even then, the lower energies of synthetic impacts are reflected in predictions $\hat{f}_{c}$ for the power spectrum's corner frequency that are even lower than those of our extension to Farin et al. (2019)'s 'thin-flow' model (see Figure 5h).

Overall, of the five models, the 'thin-flow' model described in Farin et al. (2019) best fits the results from our experiments. While the fit is imperfect, the predictions $\hat{P}_{F}^{0}$ of this model differ from the measured values $P_{F}^{0}$ by a typical factor of 2.1 , lower than that for the other models, and the model's accuracy is approximately equal across the entire range of experiments, including for the flows in the transitional regime. Constructing a statistical model for each physical model, by assuming $\ln P_{F}^{0}$ was normally distributed about $\ln \hat{P}_{F}^{0}$ with constant variance, the 'thin-flow' model is also the preferred model by the Akaike information criterion (see S9), indicating that its additional free parameter compared to the Lai et al. (2018) model is worthwhile in an information theoretic sense. This analysis did not compare models' predictions to the measured corner frequencies $f_{c}$, due to the likelihood of systematic error in the latter, but our extensions to the models of Farin et al. (2019) both predicted a trend in $\hat{f}_{c}$ consistent with measurements.

\section{Discussion}

\subsection{Velocity Profiles and the 'Thin-flow' Model}

That the 'thin-flow' model best predicts the experimental results is surprising, because we do not expect the velocity profile within the flow to be consistent with the model's assumptions. The 'thin-flow' model assumes that particles at the flow's base move across the instrumented plate's surface at approximately the flow's mean velocity, whereas previous authors suggest that the plate's roughened surface should impose a no-slip condition on the flow, in the sense that particles' velocities should tend to zero towards the flow's base (GDR MiDi, 2004; Jing et al., 2016). Furthermore, as the example of Figure 6 demonstrates, the velocity profiles we observe at the channel's wall are consistent with this no-slip condition (which we note is distinct from any micromechanical condition on rolling or sliding at particle contacts). 
a

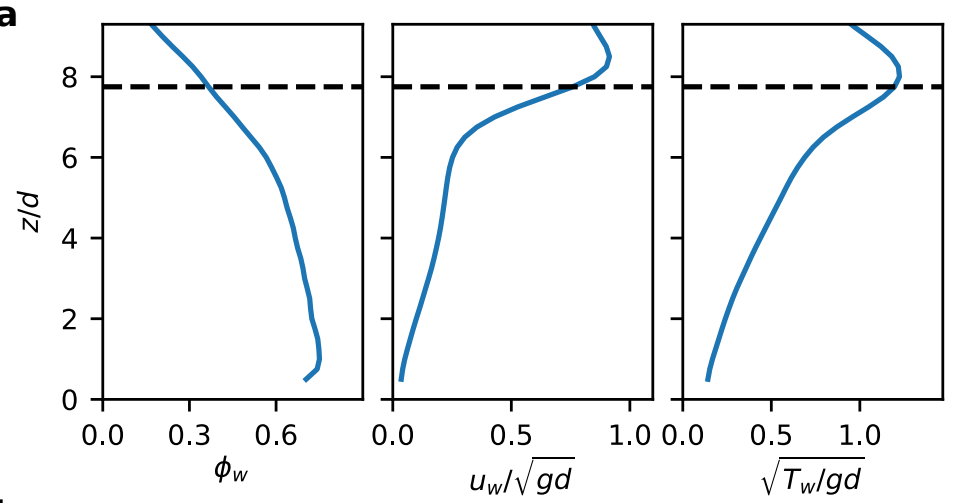

b

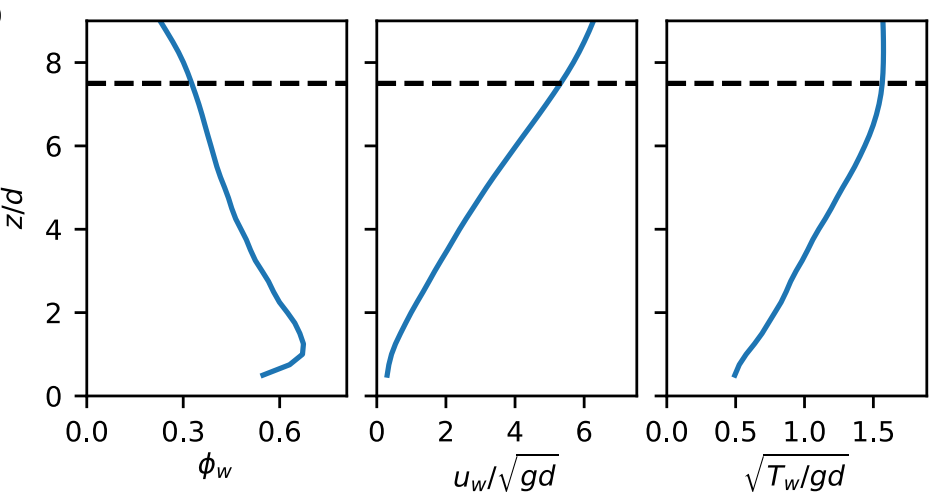

Figure 6. Examples of kinematic properties' steady profiles at the channel wall. Profiles are estimates from particle tracking velocimetry of the relative volume fraction $\phi_{w}$, the downslope velocity $u_{w}$, and the square root $\sqrt{T_{w}}$ of the granular temperature, non-dimensionalised by $\sqrt{g d}=0.14$, while the dashed lines represent the flow thicknesses $h$ inferred from the profile of $\phi_{w}$. Profiles are taken from the same experiments as for Figure 3: a) a dense flow at channel incline $\tan \theta=0.44\left(\theta=23.7^{\circ}\right)$ with release gate height $h_{g}=20 \mathrm{~mm}$; and b) a transitional-regime flow at channel incline $\tan \theta=0.52\left(\theta=27.5^{\circ}\right)$ with release gate height $h_{g}=28 \mathrm{~mm}$. 
We propose two possible explanations for the success of the 'thin-flow' model. The first is that the instrumented plate's flow-induced vibration reduces the effective friction between it and the flow, leading to basal slip and a basal flow velocity closer to the flow's mean velocity. The second is that basal particles have low velocities, but that impacts away from the flow's base make significant contributions to the basal force exerted by the flow, in such a way that the total contribution of these impacts scales with the mean velocity of the flow.

The first explanation is supported by the literature on frictional weakening and by measurements of the plate's effective friction coefficient with the flow. The reduction by vibration of a granular medium's effective friction has been documented in discrete element simulations (e.g. Capozza et al., 2009; Ferdowsi et al., 2014; Lemrich et al., 2017) and experiments (e.g. Johnson et al., 2008; Dijksman et al., 2011; Lastakowski et al., 2015), with suggestions for the necessary vibration amplitude being a particle strain of order $10^{-6}$ (Ferdowsi et al., 2014), a velocity of order $100 \mathrm{\mu m} \mathrm{s}^{-1}$ (Lastakowski et al., 2015), and an acceleration of order 0.1g (Dijksman et al., 2011). Even in the experiments in which the plate vibration amplitudes during steady flow were lowest, the plate had approximate root mean square normal displacement $10 \mathrm{~nm}$, velocity $100 \mathrm{\mu m} \mathrm{s}^{-1}$, and acceleration $20 \mathrm{~m} \mathrm{~s}^{-2}$, so a vibration-induced reduction in friction appears viable. Furthermore, the effective friction coefficients $\mu$ that we measure between the plate and the flow are too low to prevent basal slip on the surface of the plate, with Figure 7a showing that $\mu<\tan \theta$ for all channel inclines $\tan \theta$. This implies that basal particles accelerate across the plate's surface, towards the flow's mean velocity.

On the other hand, we do not directly measure any increases in velocity associated with basal slip. Over the $8 \mathrm{~cm}$ distance downslope captured by the high-speed camera, averaging over each flow's depth and each $4 \mathrm{~cm}$ half-window, the mean downslope velocities measured at the sidewall are uniform to within $10 \%$. Away from the sidewalls, Tsang et al. (2019) suggests that a granular flow will adjust to a change in basal boundary conditions over a lengthscale of order $\bar{u}^{2} / g$, for mean flow velocity $\bar{u}$ and gravitational acceleration $g$. This lengthscale varies in our experiments from $0.5 \mathrm{~mm}$ to $0.1 \mathrm{~m}$, so that we would expect the effects of any basal slip to become evident at the flow's surface within the length of the instrumented plate. However, having conducted particle image velocimetry with images captured by an overhead camera, for a flow at a channel incline $\tan \theta=$ $0.46\left(\theta=24.7^{\circ}\right)$ and with release gate height $h_{g}=20 \mathrm{~mm}$, we were unable to distin- 

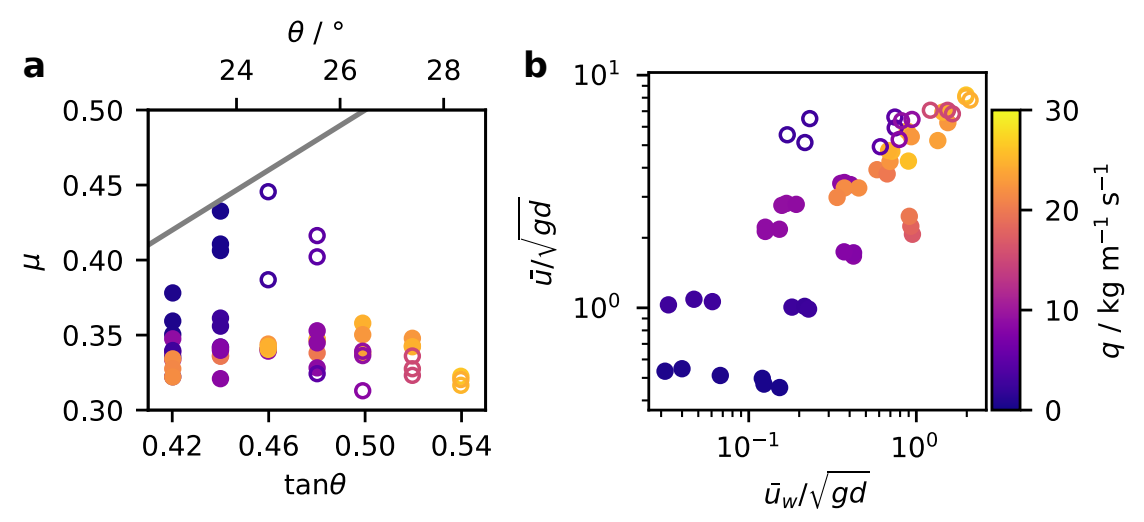

Figure 7. Discrepancies between model assumptions and measurements. a) Measurements of the effective friction coefficient $\mu$ between the instrumented plate and the flow fall consistently below the condition $\mu=\tan \theta$ for zero basal slip (grey line). b) The depth-averaged particle velocity measured at the channel wall $\bar{u}_{w}$ is poorly correlated with the mean velocity $\bar{u}$ calculated from bulk flow properties. Colours indicate each experiment's mass flux $q$ per unit channel width, and unfilled symbols represent experiments for which the flow was in the transitional regime.

guish whether the flow's surface's slight acceleration across the plate was induced by the plate, or was simply a continuation of the flow's acceleration towards a uniform state. Similarly, we attempted to detect changes in the velocity of basal particles, via Jop et al. (2005)'s method of examining soot erosion from an inserted metal plate, but our attempts were frustrated by the energetic particles' rapid erosion of soot during the insertion and removal of the plate.

Consequently, the second explanation remains feasible, with good reasons why the model of Bachelet (2018), despite being derived to describe the contributions of impacts throughout the flow's depth, might describe them less well than the 'thin-flow' model. Firstly, the Bachelet (2018) model uses profiles $u_{w}$ and $T_{w}$ that are measured at the channel's wall and may not be representative of those in the flow's interior. In fact, the mean particle velocity measured at the channel wall $\bar{u}_{w}$ correlates poorly with the mean velocity $\bar{u}$ inferred from bulk measurements (see Figure 7), while the monotonically increasing profiles $T_{w}(z)$ differ from the S-shaped profiles that previous authors propose for granular temperature profiles in the flow's interior (Hanes \& Walton, 2000; Silbert et al., 2001; Gollin et al., 2017). Secondly, Bachelet (2018) may suggest an incorrect dependence of the seismic signal on these profiles, with a particularly strong assumption being that of 
a frequency-independent attenuation constant $\gamma$. We were unable to dramatically improve the predictions of Bachelet (2018)'s model by modifying its inputs, e.g. by multiplying the profiles $u_{w}(z)$ and $\sqrt{T_{w}(z)}$ by $\bar{u} / \bar{u}_{w}$, but, under a different model for the contributions of impacts throughout the flow, such contributions could explain the relationship observed between the mean velocity $\bar{u}$ and the basal force's power spectrum $P_{F}$.

To test which explanation accounts for the success of the 'thin-flow' model, we suggest that our experimental conditions be replicated with discrete element simulations. In such simulations, a suitably roughened base could be fixed in position to prevent any vibration-induced reduction of its effective friction coefficient and any basal slip, as records of base-adjacent particles' velocities could verify. If the 'thin-flow' model continued to be accurate, then the first, 'basal slip' explanation would be disproven. Records of particle velocities throughout the flow would then permit variants of Bachelet (2018)'s model to be tested and their assumptions examined, using base-normal profiles of velocity and granular temperature measured within the flow's bulk rather than at its edge, to explain the 'thin-flow' model's accuracy. If the 'thin-flow' model were no longer accurate, however, then our first explanation would be proven and the model shown to apply only to flows with basal slip. The recorded particle velocities would then permit development of a different model, by which a small number of flow parameters could predict the seismic signal generated by flows without basal slip, analogous to the use of $\bar{u}$ in the 'thinflow' model, or of the inertial number to predict a dense granular flow's kinematic properties.

\subsection{The Inertial Number and the Seismic Signal}

Within a granular flow, the inertial number $I$ is a local, non-dimensional mean shear rate, which previous authors suggest will uniquely determine all other local, non-dimensional flow parameters (GDR MiDi, 2004; da Cruz et al., 2005; Jop et al., 2006). This ' $\mu(I)$ ' framework will not apply where a) the flow's rheology is 'non-local', in the sense that the internal stress depends on derivatives of the strain rate rather than on only the strain rate's local value (Clark \& Dijksman, 2020), or b) particles are sufficiently agitated that kinetic theory describes their motion better than a mean shear rate (Goldhirsch, 2003). If, however, the ' $\mu(I)$ ' framework applies within a two-dimensional, steady, uniform shear flow above a plate with incline $\tan \theta$, a macroscopic force balance implies that $I$ is con- 
stant and can be estimated from bulk measurements of the flow's mean velocity $\bar{u}$, volume fraction $\phi$, and depth $h$ (Jop et al., 2005), as

$$
\hat{I}=\frac{5 \bar{u} d}{2 h \sqrt{\phi g h \cos \theta}}
$$

Even if our experimental flows were uniform, without basal slip, the local inertial numbers within them will have differed significantly from $\hat{I}$, with non-locality being particularly significant within slow, thin flows; particles being particularly agitated within transitionalregime flows; and friction at the channel's walls altering the force balance. We nevertheless calculated $\hat{I}$ as a descriptor for each flow, with $\phi=\sigma / \rho h$ for flow mass per unit area $\sigma$ and particle density $\rho$ and with other quantities defined in sections 2.1 and 2.2 . We see in Figure 8a that the ' $\mu(I)$ ' framework applies for the dense experimental flows, insofar as the local, non-dimensional parameter $\tan \theta$ is closely related to $\hat{I}$.

To examine the relevance to each flow's seismic signal of this inertial number estimate $\hat{I}$, we define a non-dimensional parameter $\delta \mathcal{F}^{2}$ expressing the mean squared magnitude of high-frequency basal force fluctuations on the instrumented plate, normalised by the mean basal force. From the low-frequency amplitude $P_{F}^{0}$ and corner frequency $f_{c}$ of the basal force's power spectrum, and from gravitational acceleration $g$, inclination angle $\theta$, plate length $X$ and width $Y$, and measured mass overburden $\sigma$, we calculate for each flow

$$
\delta \mathcal{F}^{2}=\frac{2 P_{F}^{0} f_{c}}{(X Y g \sigma \cos \theta)^{2}} .
$$

To understand this definition, we recall from equation (8) that $X Y g \sigma \cos \theta$ is the mean normal force applied by the flow to the instrumented plate, over the time interval $\Delta t_{c}$ of steady flow recorded by the camera. Meanwhile, as Figure 4 indicates, $2 P_{F}^{0} f_{c}$ approximates the integral of the symmetric power spectral density $P_{F}(f)$ over all $f$ with $|f|>$ $1 \mathrm{kHz}$, this being the lowest frequency accessible to our measurements. Recalling that $\tilde{F}(f)$ is the Fourier transform over $\Delta t_{c}$ of the normal force applied to the plate, $P_{F}(f)=$ $|\tilde{F}(f)|^{2} / \Delta t_{c}$. Combining these links and then applying the Plancherel theorem (Plancherel \& Mittag-Leffler, 1910) to move to the time domain,

$$
2 P_{F}^{0} f_{c} \approx \frac{1}{\Delta t_{c}} \int_{|f|>1 \mathrm{kHz}}|\tilde{F}(f)|^{2} \mathrm{~d} f=\frac{1}{\Delta t_{c}} \int_{\Delta t_{c}}|\delta F(t)|^{2} \mathrm{~d} t,
$$

where $\delta F$ is the fluctuating normal force on the plate, high-pass-filtered above $1 \mathrm{kHz}$. Assuming that pressure fluctuations are spatially uncorrelated on the lengthscale of the plate, as discussed in $\mathrm{S} 2,2 P_{F}^{0} f_{c}$ will be proportional to the plate's area $X Y$ and $\delta \mathcal{F}^{2}$ to $1 / X Y$, 

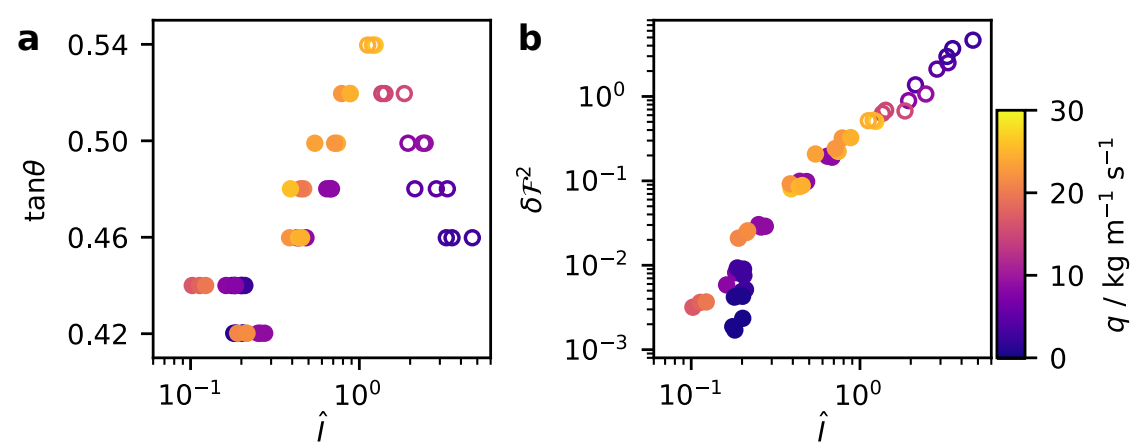

Figure 8. Relations between the inertial number $\hat{I}$ estimated from bulk flow parameters and a) the channel incline $\tan \theta, b)$ the normalised mean squared fluctuating force on the plate $\delta \mathcal{F}^{2}$. Colours indicate each experiment's mass flux $q$ per unit channel width, and unfilled symbols represent experiments for which the flow was in the transitional regime.

but $\delta \mathcal{F}^{2}$ can be thought of as a rescaling by $d^{2} / X Y$ of a local flow parameter, for mean particle diameter $d$.

Plotting $\delta \mathcal{F}^{2}$ against $\hat{I}$ for each flow, in Figure 8b, we see that this measure of the high-frequency seismic signal is well determined by the estimated inertial number. This relationship between non-dimensional, local flow parameters is in accord with the ' $\mu(I)$ ' framework, with more energetic flows producing more energetic seismic signals, even for flows to which the ' $\mu(I)$ ' framework is otherwise inapplicable.

However, it is unclear how it relates to the experimental results of Taylor and Brodsky (2017), in which the mean squared seismic accelerations generated by a torsional shear flow were directly proportional to estimates of the flow's inertial number. Whilst our results do not appear consistent with such direct proportionality, with $\delta F^{2}$ increasing much faster than $\hat{I}$, it is impossible to make a direct comparison without knowing the magnitude of the Green's function relating the accelerations discussed by Taylor and Brodsky (2017) to the forces imposed by that article's shear flow. Such Green's functions are essential in considering the seismic signal generated by a flow, and so in considering the application of our results to geophysical flows. 


\subsection{The Application of Our Results to Geophysical Flows}

Our results concern the fluctuating forces exerted by laboratory granular flows upon the base on which they travel, so their application to landquake signals necessitates consideration of two things: the Green's function that determines a flow's seismic signal from the forces it exerts, and the differences between geophysical flows' forces and those that we've studied. We limit ourselves to describing the importance of an accurate Green's function, rather than defining one, and to discussing the adjustments involved in moving from laboratory to geophysical flows, rather than validating them, but we nevertheless propose tentative links between our results and the empirical relationships observed by previous authors.

\subsubsection{The Importance of an Accurate Green's Function}

The forces exerted by a geophysical flow determine a measurable seismic signal only via a Green's function, so an accurate Green's function is necessary to interpret any landquake signal. Here, we use our experimental seismic signals to show that this is true even of certain seismic properties that previous authors have used to describe geophysical flows, including both the rate of seismic energy emission and the relative amplitudes of different landquake signals with the same source and receiver locations.

For our experiments, the Green's function appears via equation (12), which relates the basal forces exerted by the flow to the accelerations they caused and indicates that, on a larger, denser, stiffer, or more lossy plate, the same force would result in smaller accelerations and hence a smaller seismic signal. Similarly, working from the derivation of this equation in S7, the total high-frequency seismic power transferred by the flow to the plate is given in terms of the basal force's power spectral density $P_{F}$ by

$$
\Pi_{s} \approx \frac{1}{4 \sqrt{\rho_{p} H D}} \int_{1 \mathrm{kHz}}^{\infty} P_{F}(f) \mathrm{d} f
$$

dependent on plate density $\rho_{p}$, thickness $H$, and bending moment $D$. The proportion of flow energy dissipated by seismic emission is therefore a function of basal properties rather than of flow properties alone, which especially complicates comparisons between experimental and geophysical flows, such as Bachelet (2018)'s.

Furthermore, the Green's functions for seismic signals will depend differently on frequency $f$, so that basal properties will affect even the relative signal amplitudes of dif- 
ferent flows with the same Green's function. For illustration, we consider the vertical velocity response of a surficial receiver to a vertical, surficial point force, on an isotropic, homogeneous, perfectly elastic half-space with Poisson ratio 0.25 , material density $\rho_{g}$ and shear wave velocity $c_{s}$. For large source-receiver separation $r$, at leading order, Miller and Pursey (1954) showed the power spectral density $P_{v_{r}}$ of this response to be related to the power spectral density $P_{F}$ of the vertical basal force by

$$
P_{v_{r}}(f)=\frac{1.20 f^{3}}{\rho_{g}^{2} c_{s}^{5} r} P_{F}(f),
$$

which we compare to the mean velocity power spectral density over the accelerometers in our experiments,

$$
\bar{P}_{v_{j}}(f)=\frac{1}{4 \Delta t} \sum_{j=1}^{4}\left(\frac{\left|\tilde{a}_{j}(f)\right|}{2 \pi f}\right)^{2} \approx \frac{\mathcal{P} Q}{16 \pi\left(\rho_{p} H\right)^{3 / 2} X Y \sqrt{D} f} P_{F}(f) .
$$

The mean squared velocity at the receiver, being the integral of $P_{v_{r}}(f)$ over all $f$, will clearly be more sensitive to the corner frequency $f_{c}$ of $P_{F}$ than were the mean squared velocities observed in our experiments. Figure 9 shows the consequence: approximating mean squared velocities by integrating (26) and (27) between $1 \mathrm{kHz}$ and $f_{c}$, there is no constant conversion factor between the mean square velocities observed in our experiments and those that would be observed if the same flows applied the same forces in an idealised geophysical context. Even among signals with the same source and receiver locations, the Green's function determines the ratios between different signals' amplitudes, so that a signal must be properly deconvolved to infer the properties of a flow's forces.

\subsubsection{Adjustments to Forces for Geophysical Flows}

Returning to consideration of such forces, the application of our results to geophysical flows involves significant adjustments, firstly to the sizes of the flow and its constituent particles and secondly to the flow's evolution.

Clearly, geophysical flows of interest will be more extensive than our experimental flows and will involve larger particles, but these changes will not alter the underlying physics and simply necessitate adjustment of the values of flow area $A$ and particle diameter $d$ in the models of section 2.3. According to these models, a flow identical to those in our experiments, except with particles of radius $1 \mathrm{~m}$, should produce a seismic force signal with power spectral density per unit flow area $\hat{P}_{F}(f) / A$, of order $\left(10^{2}\right.$ to $\left.10^{6}\right) \mathrm{N}^{2} \mathrm{~m}^{-2} \mathrm{~s}$ below a corner frequency $f_{c}$ of order $100 \mathrm{~Hz}$. A more difficult adjustment is required to 

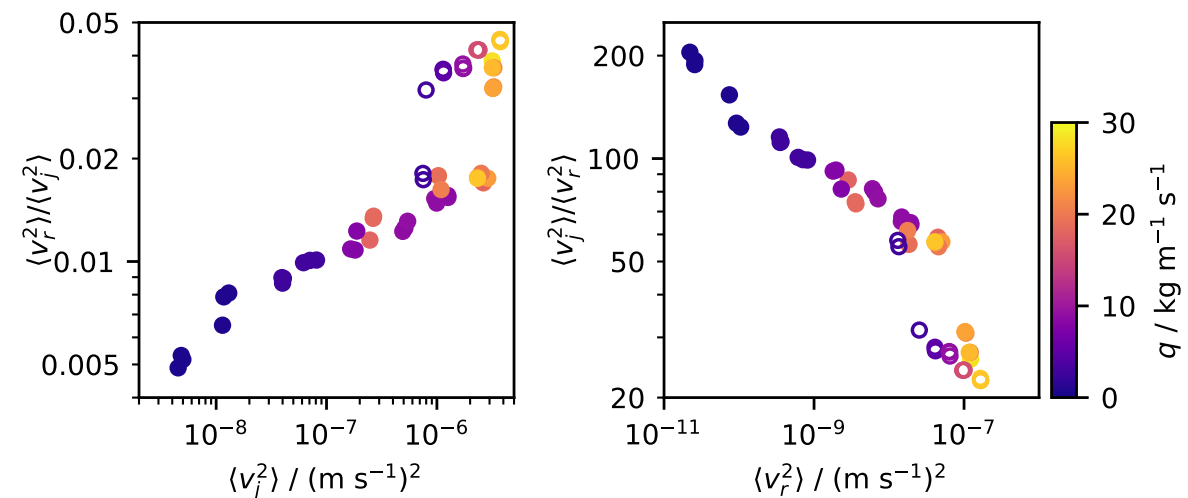

Figure 9. Comparison between the mean squared seismic velocities $\left\langle v_{j}^{2}\right\rangle$ observed in our experiments and the mean squared velocities $\left\langle v_{r}^{2}\right\rangle$ that would be observed in an idealised geophysical context. For the latter, we took $\rho_{g}=2500 \mathrm{~kg} \mathrm{~m}^{-3}, c_{s}=1 \mathrm{~km} \mathrm{~s}^{-1}$, and $r=1 \mathrm{~m}$ in equation (26), but other values would change only the prefactor. Colours indicate each experiment's mass flux $q$ per unit channel width, and unfilled symbols represent experiments for which the flow was in the transitional regime.

account for the wide particle polydispersity typical of geophysical flows (Takahashi, 1981; Nishiguchi et al., 2012), which makes $d$ hard to define and necessitates consideration of the segregation of particles by size that is well-documented within granular flows (e.g. Garve, 1925; Gray, 2018). Farin et al. (2019) proposes a promising approach for each given model, of dividing the flow into a coarse-grained front and a fine-grained tail and calculating for each a percentile of the particle size distribution that will be representative, but this proposal requires validation.

Other necessary changes relate to the flow evolution, stemming from differences in particles' coefficient of restitution and in the mechanism of their release. The glass beads in our experiments underwent collisions more elastic than are typical in geophysical flows (Kim et al., 2015), resulting in our observations of sustained saltation at relatively low channel inclinations. This implies that the precursory saltation of flow stage I, discussed in section 3.1, is unlikely to be significant for most geophysical flows, though it may be analogous to rock falls at high slope inclinations. Similarly, the energetic, saltating particles observed in the steady stage III of transitional-regime flows are likely to be rare in geophysical flows, though the coexistence of a dense core and a saltating layer is documented in snow avalanches (Pudasaini \& Hutter, 2006). In fact, the entirety of the ex- 
perimental flows' stage III is atypical of geophysical flows, since particles were released from the experimental reservoir over a long period at a constant flux, whilst the release of geophysical flows is rarely so steady or protracted. Therefore, our results should only apply to individual stages of a geophysical flow, over which flow properties vary little enough that the mean quantities we discuss are representative. Determination of quantities that are representative of an entire flow requires further work.

\subsubsection{Comparisons with Empirical Results}

Nevertheless, we can tentatively link our measurements of experimental flows' forces to the landquake signals of geophysical flows, by assuming the validity both of certain adjustments to those forces and of certain restrictions to the Green's function linking geophysical forces to landquake signals. Firstly, we assume that any precursory saltation of a geophysical flow contributes so insignificantly to the signal as to be negligible. Secondly, we suppose that the release mechanism and size distribution of geophysical particles significantly affect the signal only by determining the flow's duration and a representative diameter of its particles. Thirdly, we assume that the signal's Green's function is constant over time and corresponds to transmission along a single wave path, without significant dispersion in time. Finally, we consider the signals only at frequencies lower than any force's power spectrum's corner frequency $f_{c}$, but high enough for the stochastic impact framework and hence our results to apply.

Under these assumptions, the landquake signal $v_{r}$ between times $t_{r}$ and $t_{r}+\Delta t$ will only depend significantly on the forces exerted by the landslide between times $t_{s}$ and $t_{s}+\Delta t$, for some source-receiver delay $t_{r}-t_{s}$. Neglecting non-normal components, these forces will have a power spectral density within the relevant frequency band that is equivalent to those that we have studied and is well-described by the constant prediction $\hat{P}_{F}^{0}$ of Farin et al. (2019)'s 'thin-flow' model, for flow properties averaged between $t_{s}$ and $t_{s}+$ $\Delta t$. Writing $\tilde{G}(f)$ for the relevant frequency-space Green's function and $f_{0}$ and $f_{1}$ for the minimum and maximum frequencies under consideration, the mean squared amplitude of the signal will be

$$
\left\langle v_{r}^{2}\right\rangle_{\Delta t}=\frac{2}{\Delta t} \int_{f_{0}}^{f_{1}}\left|\tilde{v}_{r}(f)\right|^{2} \mathrm{~d} f \approx 2 \hat{P}_{F}^{0} \int_{f_{0}}^{f_{1}}|\tilde{G}(f)|^{2} \mathrm{~d} f .
$$


Given this link, we can compare our results to the empirical relations discussed in section 1.1. Qualitatively, the landquake signal's envelope will have the same shape as the envelope of the time-retarded geophysical force, as Figure 3 shows to be the case for our experimental forces and acceleration signals. Adjusting these envelopes by excluding the precursory saltation and shortening the artificially prolonged stage of steady flow, our results therefore predict the distinctive 'spindle-shaped' signal envelopes associated with geophysical granular flows (Suriñach et al., 2005). Quantitatively, our results suggest that a flow's duration will equal its signal's, as in the empirical observations of e.g. Deparis et al. (2008), though our experiments are unlike those of Farin et al. (2018) in that our release mechanism prevents comparison with the observed empirical relationship between potential energy loss and signal duration. Similarly, we cannot compare our results to the observations of e.g. Norris (1994), that the flow volume is correlated with the signal amplitude.

However, we can compare our results with other empirical relationships for the signal amplitude. Substituting equation (19) for $\hat{P}_{F}^{0}$ into equation (28) and assuming both constant particle properties and a constant Green's function, our results suggest that a flow of area $A$ in which the particle volume fraction is $\phi$ and the mean flow velocity is $\bar{u}$ will generate a signal with mean squared amplitude proportional to $\phi A \bar{u}^{3}$. Rearranging equation (22) for flow depth $h$ and noting that the mean flow momentum per unit area $q=\rho \phi h \bar{u}$, for particle density $\rho$, we recover that

$$
q^{3}=\frac{25 \rho^{3} \phi^{2} d^{2} \bar{u}^{5}}{4 \hat{I}^{2} g \cos \theta} \text { and } \bar{u}^{3}=\left(\frac{4 \hat{I}^{2} g q^{3} \cos \theta}{25 \rho^{3} \phi^{2} d^{2}}\right)^{3 / 5}
$$

for bulk inertial number $\hat{I}$, representative particle diameter $d$, gravitational acceleration $g$, and slope angle $\theta$. Among flows with constant $\hat{I}$ and $\phi$, the resulting landquake signals will therefore have root mean squared amplitude proportional to $A^{1 / 2} q^{9 / 10} \cos ^{3 / 10} \theta$. Whilst the assumption of constant $\hat{I}$ is very strong, this quantity is close to those found empirically to be approximately proportional to landquake signal amplitude: the work rate against friction used by e.g. Schneider et al. (2010), which will be equal to $\mu A q \cos \theta$ for basal friction coefficient $\mu$, and the total flow momentum used by e.g. Hibert et al. (2015), equal to $A q$. Holding all else constant, the scalings $A^{1 / 2}$ and $A$ correspond to spatially separated impacts' signals being perfectly uncorrelated and perfectly correlated, respectively, so $\mathrm{S} 2$ suggests that $A^{1 / 2}$ is likely to be a better approximation, while the scalings $q^{9 / 10}$ and $q$ are unlikely to be distinguishable in the field. 


\section{Conclusion}

In conclusion, our experimental apparatus and data analysis permitted us to study the normal force exerted by a granular flow upon the base over which it travels, by measuring its high-frequency power spectral density and testing a range of existing models that predict this spectral density from the flow's properties. Figure 5 shows the 'thinflow' model of Farin et al. (2019) to best predict the spectral density at frequencies well below its corner frequency and demonstrates that our extension of that model to higher frequencies, using Hertz theory, correctly predicts the corner frequency's behaviour. We've proposed that the success of the 'thin-flow' model, despite our experimental flows' thickness compared to their consituent particles, can be explained either by slip at each flow's base or by the contributions to the seismic signal of impacts throughout each flow's depth, and we've discussed the adjustments required to apply our results to the landquake signals generated by the forces of geophysical granular flows. Making such adjustments, under certain restrictive assumptions, the 'thin-flow' model's predictions are consistent with the empirical observation that a landquake signal's amplitude is approximately proportional to the momentum per unit area of the flow region that generated it.

Finally, our results are also relevant to two open questions on geophysical granular flows' dynamics: 1) the relation between the mean and fluctuating forces exerted by a flow; and 2) the low values of effective friction inferred for many geophysical flows. On the first question, previous authors have suggested that the typical magnitude of fluctuations is proportional to the magnitude of the mean force (McCoy et al., 2013; Hsu et al., 2014), but we show in Figure $8 \mathrm{~b}$ that the ratio between the two, $\delta \mathcal{F}$, varies over two orders of magnitude between our experimental flows, dependent on a bulk inertial number. On the second, acoustic fluidisation is one of many possible explanations suggested for the low effective friction necessary to explain many geophysical flows' long runouts (Davies, 1982; Lucas et al., 2014), but we are not aware of it having been previously demonstrated without the application of external forcing. As Figure 7a illustrates, our measurements of $\mu$ show the effective friction taking values on the plate lower than the channel incline $\tan \theta$, which is implied to be its approximate off-plate value by both the downslope uniformity of the flow at the sidewalls and the saturation of flow velocity observed at the surface. Since the base's roughness is identical in each location, we believe it possible that this reduced friction is associated with the strong acoustic vibrations of the plate, induced by the flow itself. 


\section{Acknowledgments}

This work was primarily funded by the ERC project SLIDEQUAKES, with supporting funds from La Société des Amis de l'ESPCI and from IPGP. The authors are aware of no conflict of interest. The experimental data are available at the Pangaea repository [In progress]. We are grateful to Abdelhak Souilah for his construction of the experimental apparatus.

\section{References}

Akaike, H. (1971). Determination of the number of factors by an extended maximum likelihood principle (Tech. Rep.). Inst. Statist. Math.

Akaike, H. (1974). A new look at the statistical model identification. IEEE Transactions on Automatic Control, 19(6), 716-723. Retrieved from https://link .springer. com/content/pdf/10.1007\%2F978-1-4612-1694-0.pdf

Akaike, H. (1978). On the likelihood of a time series model. Journal of the Royal Statistical Society. Series D (The Statistician), 27(3/4), 217-235. Retrieved from http://www.jstor.org/stable/2988185

Allstadt, K. (2013). Extracting source characteristics and dynamics of the August 2010 Mount Meager landslide from broadband seismograms. Journal of Geophysical Research: Earth Surface, 118(3), 1472-1490. Retrieved from https:// agupubs.onlinelibrary.wiley.com/doi/abs/10.1002/jgrf.20110 doi: 10 $.1002 /$ jgrf.20110

Arran, M. I., Mangeney, A., de Rosny, J., Farin, M., Toussaint, R., \& Roche, O. (2020). Laboratory landquakes (Vol. (In progress)).

Babic, M. (1997). Average balance equations for granular materials. International Journal of Engineering Science, 35(5), 523-548. Retrieved from http://www.sciencedirect.com/science/article/pii/S0020722596000948 doi: 10.1016/S0020-7225(96)00094-8

Bachelet, V. (2018). Étude expérimentale des émissions acoustiques générées par les écoulements granulaires (Unpublished doctoral dissertation). Institut de Physique du Globe de Paris.

Berrocal, J., Espinosa, A., \& Galdos, J. (1978). Seismological and geological aspects of the Mantaro landslide in Peru. Nature, 275, 553-536.

Börzsönyi, T., \& Ecke, R. E. $\quad$ (2006, December). Rapid granular flows on a rough 
incline: phase diagram, gas transition, and effects of air drag. Physical Review E, 74(6 Pt 1), 061301. Retrieved from https://doi.org/10.1103/PhysRevE .74 .061301 doi: 10.1103/physreve.74.061301

Brodsky, E. E., Gordeev, E., \& Kanamori, H. (2003). Landslide basal friction as measured by seismic waves. Geophysical Research Letters, 30(24). Retrieved from https://agupubs.onlinelibrary.wiley.com/doi/abs/10.1029/ 2003GL018485 doi: 10.1029/2003GL018485

Capozza, R., Vanossi, A., Vezzani, A., \& Zapperi, S. (2009). Suppression of friction by mechanical vibrations. Physical Review Letters, 103, 085502. doi: 10.1103/ PhysRevLett.103.085502

Chao, W.-A., Wu, Y.-M., Zhao, L., Chen, H., Chen, Y.-G., Chang, J.-M., \& Lin, C.-M. (2017). A first near real-time seismology-based landquake monitoring system. Scientific Reports, 7, 43510. doi: 10.1038/srep43510

Ciarlet, P. G. (1997). Chapter 1 - Linearly Elastic Plates. In P. G. Ciarlet (Ed.), Mathematical Elasticity (Vol. 27, pp. 3-127). Elsevier. Retrieved from http:// www.sciencedirect.com/science/article/pii/S0168202497800070 doi: 10 $.1016 /$ S0168-2024(97)80007-0

Clark, A. H., \& Dijksman, J. A. (2020). Editorial: Non-local modeling and diverging lengthscales in structured fluids. Frontiers in Physics, 8, 18. Retrieved from https://www.frontiersin.org/article/10.3389/fphy.2020.00018 doi: 10 $.3389 /$ fphy.2020.00018

Coe, J. A., Baum, R. L., Allstadt, K. E., Kochevar, J., Bernard F., Schmitt, R. G., Morgan, M. L., ... Kean, J. W. ～(2016). Rock-avalanche dynamics revealed by large-scale field mapping and seismic signals at a highly mobile avalanche in the West Salt Creek valley, western Colorado. Geosphere, 12(2), 607-631. Retrieved from https://doi.org/10.1130/GES01265.1 doi: 10.1130/GES01265.1

Cuomo, S. (2020). Modelling of flowslides and debris avalanches in natural and engineered slopes: a review. Geoenvironmental Disasters, 7(1).

da Cruz, F., Emam, S., Prochnow, M., Roux, J.-N., \& Chevoir, F. (2005). Rheophysics of dense granular materials: Discrete simulation of plane shear flows. Physical Review E, 72, 021309. Retrieved from https://link.aps.org/doi/ 10.1103/PhysRevE.72.021309 doi: 10.1103/PhysRevE.72.021309 
Dahlen, F. A. (1993). Single-force representation of shallow landslide sources. Bulletin of the Seismological Society of America, 83(1), 130. Retrieved from http://dx.doi.org/

Dammeier, F., Moore, J. R., Hammer, C., Haslinger, F., \& Loew, S. (2016). Automatic detection of alpine rockslides in continuous seismic data using hidden Markov models. Journal of Geophysical Research: Earth Surface, 121(2), 351371. Retrieved from https://agupubs.onlinelibrary.wiley.com/doi/abs/ 10.1002/2015JF003647 doi: 10.1002/2015JF003647

Dammeier, F., Moore, J. R., Haslinger, F., \& Loew, S. ～(2011). Characterization of alpine rockslides using statistical analysis of seismic signals. Journal of Geophysical Research: Earth Surface, 116(F4). Retrieved from https://agupubs .onlinelibrary.wiley.com/doi/abs/10.1029/2011JF002037 doi: 10.1029/ 2011JF002037

Davies, T. R. H. (1982). Spreading of rock avalanche debris by mechanical fluidization. Rock mechanics, 15, 9-24.

Delannay, R., Valance, A., Mangeney, A., Roche, O., \& Richard, P. (2017). Granular and particle-laden flows: from laboratory experiments to field observations. Journal of Physics D: Applied Physics, 50(5), 053001. Retrieved from https ://doi.org/10.1088\%2F1361-6463\%2F50\%2F5\%2F053001 doi: $10.1088 / 1361-6463 / 50 / 5 / 053001$

Deparis, J., Jongmans, D., Cotton, F., Baillet, L., Thouvenot, F., \& Hantz, D. (2008). Analysis of rock-fall and rock-fall avalanche seismograms in the French Alps. Bulletin of the Seismological Society of America, 98(4), 1781-1796. Retrieved from https://doi.org/10.1785/0120070082 doi: $10.1785 / 0120070082$

Dijksman, J. A., Wortel, G. H., van Dellen, L. T. H., Dauchot, O., \& van Hecke, M. (2011). Jamming, yielding, and rheology of weakly vibrated granular media. Physical Review Letters, 10\%, $108303 . \quad$ Retrieved from https://link.aps.org/doi/10.1103/PhysRevLett.107.108303 doi: 10.1103/PhysRevLett.107.108303

Eissler, H., \& Kanamori, H. (1987). A single-force model for the 1975 Kalapana, Hawaii earthquake. Journal of Geophysical Research, 92, 4827-4836. doi: 10 .1029/JB092iB06p04827 
Ekström, G., \& Stark, C. P. (2013). Simple scaling of catastrophic landslide dynamics. Science, 339(6126), 1416-1419. Retrieved from http://science .sciencemag.org/content/339/6126/1416 doi: 10.1126/science.1232887

Farin, M., Mangeney, A., de Rosny, J., Toussaint, R., \& Trinh, P.-T.

(2018) Link between the dynamics of granular flows and the generated seismic signal: Insights from laboratory experiments.

Journal of Geophysical Research: Earth Surface, 123(6), 1407-1429.

Retrieved from https:// agupubs.onlinelibrary.wiley.com/doi/abs/10.1029/2017JF004296 doi: 10.1029/2017JF004296

Farin, M., Tsai, V. C., Lamb, M. P., \& Allstadt, K. E. $\quad$ (2019). A physical model of the high-frequency seismic signal generated by debris flows. Earth Surface Processes and Landforms, 44(13), 2529-2543. Retrieved from https://onlinelibrary.wiley.com/doi/abs/10.1002/esp.4677 doi: $10.1002 /$ esp. 4677

Favreau, P., Mangeney, A., Lucas, A., Crosta, G., \& Bouchut, F. (2010). Numerical modeling of landquakes. Geophysical Research Letters, 37(15). Retrieved from https://agupubs .onlinelibrary.wiley.com/doi/abs/10.1029/ 2010GL043512 doi: 10.1029/2010GL043512

Ferdowsi, B., Griffa, M., Guyer, R., Johnson, P., \& Carmeliet, J. ～(2014). $\quad$ Effect of boundary vibration on the frictional behavior of a dense sheared granular layer. Acta Mechanica, 225, 2227-2237.

Fleming, R., Taylor, F., \& (U.S.), G. S. (1980). Estimating the costs of landslide damage in the United States. U.S. Department of the Interior, Geological Survey. Retrieved from https://books.google.fr/books?id=DGnlhwP6h8cC

Froude, M. J., \& Petley, D. N. (2018). Global fatal landslide occurrence from 2004 to 2016. Nat. Hazards Earth Syst. Sci., 18, 2161-2181.

Fuchs, F., Lenhardt, W., Bokelmann, G., \& the AlpArray Working Group. (2018). Seismic detection of rockslides at regional scale: examples from the Eastern Alps and feasibility of kurtosis-based event location. Earth Surface Dynamics, 6. doi: 10.5194/esurf-6-955-2018

Fukao, Y. (1995). Single-force representation of earthquakes due to landslides or the collapse of caverns. Geophysical Journal International, 122(1), 243-248. Retrieved from https://doi.org/10.1111/j.1365-246X.1995.tb03551.x doi: 

10.1111/j.1365-246X.1995.tb03551.x

Galitzine (Golitsyn), B. (1915). Sur le tremblement de terre du 18 février 1911. Comptes rendus hebdomadaires des séances de l'Académie des sciences, 160, 810-813.

Garve, T. W. (1925). Segregation in bins. J. Amer. Ceram. Soc., 80, 666.

GDR MiDi. $\quad(2004,01)$. On dense granular flows. The European Physical Journal E, 14(4), 341-365. Retrieved from https://doi.org/10.1140/epje/ i2003-10153-0 doi: 10.1140/epje/i2003-10153-0

Gimbert, F., \& Tsai, V. C. (2015). Predicting short-period, wind-wave-generated seismic noise in coastal regions. Earth and Planetary Science Letters, 426, 280-292. Retrieved from http://www.sciencedirect.com/science/article/ pii/S0012821X15003738 doi: 10.1016/j.epsl.2015.06.017

Given, D. D., Allen, R. M., Baltay, A. S., Bodin, P., Cochran, E. S., Creager, K., ... Yelin, T. S. (2018). Revised technical implementation plan for the ShakeAlert system: An earthquake early warning system for the West Coast of the United States (Tech. Rep.). $\quad$ U.S. Geological Survey Open-File Report. doi: $10.3133 /$ ofr 20181155

Goldhirsch, I. (2003). Rapid granular flows. Annual Review of Fluid Mechanics, 35(1), 267-293. Retrieved from https://doi.org/10.1146/annurev.fluid .35 .101101 .161114 doi: 10.1146/annurev.fluid.35.101101.161114

Gollin, D., Berzi, D., \& Bowman, E. (2017). Extended kinetic theory applied to inclined granular flows: role of boundaries. Granular Matter, 19(56).

Gray, J. M. N. T. (2018). Particle Segregation in Dense Granular Flows. Annual Review of Fluid Mechanics, 50(1), 407-433. Retrieved from https://doi.org/ 10.1146/annurev-fluid-122316-045201 doi: 10.1146/annurev-fluid-122316 $-045201$

Guzzetti, F., Gariano, S. L., Peruccacci, S., Brunetti, M. T., Marchesini, I., Rossi, M., \& Melillo, M. (2020). Geographical landslide early warning systems. $\quad$ Earth-Science Reviews, 200, 102973. Retrieved from http://www.sciencedirect.com/science/article/pii/S0012825219304635 doi: $10.1016 /$ j.earscirev.2019.102973

Hanes, D. M., \& Walton, O. R. (2000). Simulations and physical measurements of glass spheres flowing down a bumpy incline. Powder Technology, 109(1), 133- 
144. Retrieved from http://www.sciencedirect.com/science/article/pii/ S0032591099002326 doi: 10.1016/S0032-5910(99)00232-6

Hasegawa, H. S., \& Kanamori, H. (1987). Source mechanism of the magnitude 7.2 Grand Banks earthquake of November 1929: Double couple or submarine landslide? Bulletin of the Seismological Society of America, $77(6), 1984$. Retrieved from http://dx.doi.org/

Hertz, H. R. (1881). Über die berührung fester elastischer körper. Journal für die reine und angewandte Mathematik, 92, 156-171. Retrieved from https://home .uni-leipzig.de/pwm/web/download/Hertz1881.pdf

Hervás, J. E. (2003). Lessons learnt from landslide disasters in Europe. eur 20558 en (Tech. Rep.). Ispra, Italy: European Commission.

Hibert, C., Ekström, G., \& Stark, C. P. (2017). The relationship between bulk-mass momentum and short-period seismic radiation in catastrophic landslides. Journal of Geophysical Research: Earth Surface, 122(5), 1201-1215. Retrieved from https://agupubs.onlinelibrary.wiley.com/doi/abs/10.1002/ 2016JF004027 doi: 10.1002/2016JF004027

Hibert, C., Mangeney, A., Grandjean, G., Baillard, C., Rivet, D., Shapiro, N. M., ... Crawford, W. (2014). Automated identification, location, and volume estimation of rockfalls at Piton de la Fournaise volcano. Journal of Geophysical Research: Earth Surface, 119(5), 1082-1105. Retrieved from https:// agupubs .onlinelibrary .wiley.com/doi/abs/10.1002/2013JF002970 doi: 10.1002/2013JF002970

Hibert, C., Mangeney, A., Grandjean, G., \& Shapiro, N. M. ～(2011). Slope instabilities in Dolomieu crater, Réunion Island: From seismic signals to rockfall characteristics. Journal of Geophysical Research: Earth Surface, 116(F4). doi: 10.1029/2011JF002038

Hibert, C., Stark, C. P., \& Ekström, G. ～(2015). Dynamics of the Oso-Steelhead landslide from broadband seismic analysis. Natural Hazards and Earth System Sciences, 15(6), 1265-1273. Retrieved from https://www.nat-hazards-earth -syst-sci.net/15/1265/2015/ doi: 10.5194/nhess-15-1265-2015

Hsu, L., Dietrich, W. E., \& Sklar, L. S. (2014). Mean and fluctuating basal forces generated by granular flows: Laboratory observations in a large vertically rotating drum. Journal of Geophysical Research: Earth Surface, 119(6), 1283- 
1309. Retrieved from https://agupubs.onlinelibrary.wiley.com/doi/abs/ 10.1002/2013JF003078 doi: 10.1002/2013JF003078

Hungr, O., \& Morgenstern, N. R. (1984). Experiments on the flow behaviour of granular materials at high velocity in an open channel. Géotechnique, 34(3), 405-413. Retrieved from https://doi.org/10.1680/geot.1984.34.3.405 doi: 10.1680 /geot.1984.34.3.405

Hurvich, C. M., \& Tsai, C.-L. (1989). Regression and time series model selection in small samples. Biometrika, 76(2), 297-307. Retrieved from https://doi.org/ 10.1093/biomet/76.2.297 doi: 10.1093/biomet/76.2.297

Jeffreys, H. (1923). The Pamir earthquake of 1911 February 18, in relation to the depths of earthquake foci. Geophysical Journal International, 1(s2), 22-31. Retrieved from https://onlinelibrary.wiley.com/doi/abs/10.1111/ j.1365-246X.1923.tb06566. $\mathrm{x}$ doi: 10.1111/j.1365-246X.1923.tb06566.x

Jing, L., Kwok, C. Y., Leung, Y. F., \& Sobral, Y. D. (2016). Characterization of base roughness for granular chute flows. Physical Review E, 94, 052901. Retrieved from https://link.aps.org/doi/10.1103/PhysRevE.94.052901 doi: 10.1103/PhysRevE.94.052901

John Steel. (2019). Fiche technique acier s355 (Tech. Rep.). Author. Retrieved from https://www.john-steel.com/fr/acier/29-plaque-d-acier-decape -et-graisse.html

Johnson, P. A., Savage, H., Knuth, M., Gomberg, J., \& Marone, C. (2008). Effects of acoustic waves on stick-slip in granular media and implications for earthquakes. Nature, 451, 57-60.

Jop, P., Forterre, Y., \& Pouliquen, O. (2005). Crucial role of sidewalls in granular surface flows: consequences for the rheology. Journal of Fluid Mechanics, 541, 167-192. doi: 10.1017/S0022112005005987

Jop, P., Forterre, Y., \& Pouliquen, O. (2006). A constitutive law for dense granular flows. Nature, 441(7094), 727-730. Retrieved from http://www. nature .com/nature/journal/v441/n7094/suppinfo/nature04801\_S1.html (10.1038/nature04801) doi: 10.1038/nature04801

Kanamori, H., \& Given, J. W. (1982). Analysis of long-period seismic waves excited by the May 18, 1980, eruption of Mount St. Helens - A terrestrial monopole? Journal of Geophysical Research: Solid Earth, 87(B7), 5422-5432. Retrieved 
from https://agupubs.onlinelibrary.wiley.com/doi/abs/10.1029/ JB087iB07p05422 doi: 10.1029/JB087iB07p05422

Kawakatsu, H. (1989). Centroid single force inversion of seismic waves generated by landslides. Journal of Geophysical Research: Solid Earth, 94(B9), 1236312374. Retrieved from https://agupubs.onlinelibrary.wiley.com/doi/ abs/10.1029/JB094iB09p12363 doi: 10.1029/JB094iB09p12363

Kean, J. W., Coe, J. A., Coviello, V., Smith, J. B., McCoy, S. W., \& Arattano, M. (2015). Estimating rates of debris flow entrainment from ground vibrations. Geophysical Research Letters, 42(15), 6365-6372. Retrieved from https:// agupubs.onlinelibrary.wiley.com/doi/abs/10.1002/2015GL064811 doi: 10.1002/2015GL064811

Kim, D. H., Gratchev, I., Berends, J., \& Balasubramaniam, A. (2015). Calibration of restitution coefficients using rockfall simulations based on 3d photogrammetry model: a case study. Natural Hazards, 78, 1931-1946. Retrieved from https://doi.org/10.1007/s11069-015-1811-x

Kullback, S., \& Leibler, R. A. (1951). On information and sufficiency. Ann. Math. Statist., 22(1), 79-86. Retrieved from https://doi.org/10.1214/aoms/ 1177729694 doi: 10.1214/aoms/1177729694

La Rocca, M., Galluzzo, D., Saccorotti, G., Tinti, S., Cimini, G. B., \& Del Pezzo, E. (2004). Seismic signals associated with landslides and with a tsunami at Stromboli volcano, Italy. Bulletin of the Seismological Society of America, 94(5), 1850-1867. Retrieved from https://doi.org/10.1785/012003238 doi: $10.1785 / 012003238$

Lai, V. H., Tsai, V. C., Lamb, M. P., Ulizio, T. P., \& Beer, A. R. (2018). The seismic signature of debris flows: Flow mechanics and early warning at Montecito, California. $\quad$ Geophysical Research Letters, 45(11), p5528-5535. doi: 10.1029/2018GL077683

Lastakowski, H., Géminard, J.-C., \& Vidal, V. (2015). Granular friction: Triggering large events with small vibrations. Scientific Reports, 5, 13455.

Lee, E.-J., Liao, W.-Y., Lin, G.-W., Chen, P., Mu, D., \& Lin, C.-W. (2019). Towards automated real-time detection and location of large-scale landslides through seismic waveform back projection. Geofluids. doi: 10.1155/2019/ 1426019 
Lemrich, L., Carmeliet, J., Johnson, P. A., Guyer, R., \& Jia, X. (2017). Dynamic induced softening in frictional granular materials investigated by discreteelement-method simulation. Physical Review E, 96, 062901. Retrieved from https://link.aps.org/doi/10.1103/PhysRevE.96.062901 doi: 10.1103/PhysRevE.96.062901

Levy, C., Mangeney, A., Bonilla, F., Hibert, C., Calder, E. S., \& Smith, P. J. (2015). Friction weakening in granular flows deduced from seismic records at the Soufrière Hills volcano, Montserrat. Journal of Geophysical Research: Solid Earth, 120(11), 7536-7557. Retrieved from https://agupubs.onlinelibrary .wiley.com/doi/abs/10.1002/2015JB012151 doi: 10.1002/2015JB012151

Lin, C.-H., Kumagai, H., Ando, M., \& Shin, T. (2010). Detection of landslides and submarine slumps using broadband seismic networks. Geophysical Research Letters, 37. doi: 10.1029/2010GL044685

Love, A. E. H., \& Darwin, G. H. (1888). XVI. the small free vibrations and deformation of a thin elastic shell. Philosophical Transactions of the Royal Society of London. (A.), 179, 491-546. Retrieved from https:// royalsocietypublishing.org/doi/abs/10.1098/rsta.1888.0016 doi: $10.1098 /$ rsta. 1888.0016

Lucas, A., Mangeney, A., \& Ampuero, J. P. (2014). Frictional velocity-weakening in landslides on Earth and on other planetary bodies. Nature Communications, 5, 3417. doi: 10.1038/ncomms4417

McCoy, S. W., Tucker, G. E., Kean, J. W., \& Coe, J. A. $\quad$ (2013). Field measurement of basal forces generated by erosive debris flows. Geophysical Research: Earth Surface, 118(2), 589-602. Retrieved from https://agupubs.onlinelibrary.wiley.com/doi/abs/10.1002/jgrf . 20041 doi: $10.1002 /$ jgrf.20041

McLaskey, G. C., \& Glaser, S. D. (2010). Hertzian impact: Experimental study of the force pulse and resulting stress waves. The Journal of the Acoustical Society of America, 128(3), 1087-1096. Retrieved from https://asa.scitation . org/doi/abs/10.1121/1.3466847 doi: 10.1121/1.3466847

Michlmayr, G., \& Or, D. $\quad(2014,01)$ Mechanisms for acoustic emissions generation during granular shearing. Granular Matter, 16(5), 627-640. Retrieved from https://doi.org/10.1007/s10035-014-0516-2 doi: 
Miller, G. F., \& Pursey, H. (1954). The Field and Radiation Impedance of Mechanical Radiators on the Free Surface of a Semi-Infinite Isotropic Solid. Proceedings of the Royal Society of London A: Mathematical, Physical and Engineering Sciences, 223(1155), 521-541. Retrieved from http://rspa.royalsocietypublishing.org/content/223/1155/521 doi: 10.1098/rspa.1954.0134

Moretti, L., Allstadt, K., Mangeney, A., Capdeville, Y., Stutzmann, E., \& Bouchut, F. (2015). Numerical modeling of the Mount Meager landslide constrained by its force history derived from seismic data. Journal of Geophysical Research: Solid Earth, 120(4), 2579-2599. Retrieved from https:// agupubs.onlinelibrary.wiley.com/doi/abs/10.1002/2014JB011426 doi: 10.1002/2014JB011426

Moretti, L., Mangeney, A., Capdeville, Y., Stutzmann, E., Huggel, C., Schneider, D., \& Bouchut, F. (2012). Numerical modeling of the Mount Steller landslide flow history and of the generated long period seismic waves. Geophysical Research Letters, 39(16). Retrieved from https://agupubs.onlinelibrary.wiley .com/doi/abs/10.1029/2012GL052511 doi: 10.1029/2012GL052511

Moretti, L., Mangeney, A., Walter, F., Capdeville, Y., Bodin, T., Stutzmann, E., \& Le Friant, A. (2020). Constraining landslide characteristics with Bayesian inversion of field and seismic data. Geophysical Journal International. Retrieved from https://doi.org/10.1093/gji/ggaa056 (ggaa056) doi: $10.1093 /$ gji/ggaa056

National Research Council. (1985). Reducing losses from landsliding in the united states. The National Academies Press.

Nishiguchi, Y., Uchida, T., Takezawa, N., Ishizuka, T., \& Mizuyama, T. Runout characteristics and grain size distribution of large-scale debris flows triggered by deep catastrophic landslides. International Journal of Erosion Control Engineering, 5(1), 16-26. doi: 10.13101/ijece.5.16

Norris, R. D. (1994). Seismicity of rockfalls and avalanches at three Cascade range volcanoes: Implications for seismic detection of hazardous mass movements. Bulletin of the Seismological Society of America, 84(6), 1925. Retrieved from http://dx.doi.org/ 
Peterschmitt, É., \& de Visintini, G. (1964). Sur les enregistrements séismiques associés à la catastrophe de la digue de Vajont du 9 octobre 1963. Comptes rendus hebdomadaires des séances de l'Académie des sciences, 258, 2629-2632.

Plancherel, M., \& Mittag-Leffler, G. $\quad$ (1910). Contribution à l'étude de la représentation d'une fonction arbitraire par les intégrales définies. Rendiconti del Circolo Matematico di Palermo, 30(1), 289-335.

Pudasaini, S., \& Hutter, K. (2006). Avalanche dynamics: Dynamics of rapid flows of dense granular avalanches. Springer. Retrieved from https://books.google .co.uk/books?id=gri6NAEACAAJ

Roche, O., van den Wildenberg, S., Valance, A., Delannay, R., Mangeney, A., Corna, L., \& Latchimy, T. (2020). Measurement of effective friction at the base of granular flows in an inclined channel. Physical Review E, (Under review).

Schneider, D., Bartelt, P., Caplan-Auerbach, J., Christen, M., Huggel, C., \& McArdell, B. W. (2010). Insights into rock-ice avalanche dynamics by combined analysis of seismic recordings and a numerical avalanche model. Journal of Geophysical Research: Earth Surface, 115(F4). Retrieved from https:// agupubs.onlinelibrary.wiley.com/doi/abs/10.1029/2010JF001734 doi: 10.1029/2010JF001734

Scholl, H. J., Ballard, S., Carnes, S., Herman, A., \& Parker, N. (2017). Informational challenges in early disaster response: The massive Oso/SR530 landslide 2014 as case in point. Proceedings of the 50th Hawaii International Conference on System Sciences.

Schuster, R. L., \& Fleming, R. W. (1986). Economic losses and fatalities due to landslides. Bulletin of the Association of Engineering Geologists, 23(1), p1128.

Sigmund Lindner. (2018). Product data sheet. Retrieved online. Retrieved from https://www.sigmund-lindner.com/en/products/glass-beads/ dispersing-beads/

Silbert, L. E., Ertaş, D., Grest, G. S., Halsey, T. C., Levine, D., \& Plimpton, S. J. (2001). Granular flow down an inclined plane: Bagnold scaling and rheology. Physical Review E, 64, 051302. Retrieved from https://link.aps.org/doi/ 10.1103/PhysRevE.64.051302 doi: 10.1103/PhysRevE.64.051302

Steel SS. (2019). Data table for carbon steel s355mc (Tech. Rep.). Author. Retrieved 
from https://www.steelss.com/Carbon-steel/s355mc.html

Suriñach, E., Vilajosana, I., Khazaradze, G., Biescas, B., Furdada, G., \& Vilaplana, J. M. (2005). Seismic detection and characterization of landslides and other mass movements. Natural Hazards and Earth System Sciences, 5(6), 791-798. Retrieved from https://www.nat-hazards-earth-syst-sci.net/5/791/ 2005/ doi: 10.5194/nhess-5-791-2005

Taberlet, N., Richard, P., Jenkins, J. T., \& Delannay, R. (2007). Density inversion in rapid granular flows: the supported regime. Eur. Phys. J. E, 22(1), 17-24. Retrieved from https://doi.org/10.1140/epje/e2007-00010-5 doi: 10.1140/ epje/e2007-00010-5

Takahashi, T. (1981). Debris flow. Annual Review of Fluid Mechanics, 13(1), 57-77. Retrieved from https://doi.org/10.1146/annurev.fl.13.010181.000421 doi: 10.1146/annurev.fl.13.010181.000421

Taylor, S., \& Brodsky, E. E. (2017). Granular temperature measured experimentally in a shear flow by acoustic energy. Physical Review E, 96, 032913. Retrieved from https://link.aps.org/doi/10.1103/PhysRevE.96.032913 doi: 10.1103/PhysRevE.96.032913

Tsai, V. C., \& Atiganyanun, S. (2014). Green's functions for surface waves in a generic velocity structure. Bulletin of the Seismological Society of America, 104(5), 2573-2578. Retrieved from https://doi.org/10.1785/0120140121 doi: $10.1785 / 0120140121$

Tsai, V. C., Minchew, B., Lamb, M. P., \& Ampuero, J.-P. (2012). A physical model for seismic noise generation from sediment transport in rivers. Geophysical Research Letters, 39(2). Retrieved from https://agupubs.onlinelibrary .wiley.com/doi/abs/10.1029/2011GL050255 doi: 10.1029/2011GL050255

Tsang, J. M. F., Dalziel, S. B., \& Vriend, N. M. (2019). The granular Blasius problem. Journal of Fluid Mechanics, 872, 784-817. doi: 10.1017/jfm.2019.357

van Asch, T., Malet, J., van Beek, L., \& Amitrano, D. (2007). Techniques, advances, problems and issues in numerical modelling of landslide hazard. Bulletin de la Société Géologique de France, 178(2), p65-88.

Weichert, D., Horner, R. B., \& Evans, S. G. (1994). Seismic signatures of landslides: The 1990 Brenda Mine collapse and the 1965 Hope rockslides. Bulletin of the Seismological Society of America, 84 (5), 1523-1532. 
Yamada, M., Kumagai, H., Matsushi, Y., \& Matsuzawa, T.

(2013). Dynamic landslide processes revealed by broadband seismic records.

Geophysical Research Letters, 40(12), 2998-3002.

Retrieved from https:// agupubs.onlinelibrary.wiley.com/doi/abs/10.1002/grl.50437 doi: $10.1002 / \operatorname{grl} .50437$

Yamada, M., Mangeney, A., Matsushi, Y., \& Matsuzawa, T. (2018). Estimation of dynamic friction and movement history of large landslides. Landslides, 15, 1963-1974. doi: 10.1007/s10346-018-1002-4

Yamada, M., Mangeney, A., Matsushi, Y., \& Moretti, L. (2016). Estimation of dynamic friction of the Akatani landslide from seismic waveform inversion and numerical simulation. Geophysical Journal International, 206(3), 1479-1486. Retrieved from https://doi.org/10.1093/gji/ggw216 doi: $10.1093 /$ gji $/ g g w 216$

Zhao, J., Ouyang, C. J., Ni, S. D., Chu, R. S., \& Mangeney, A. (2020). Analysis of the June 2017 Maoxian Landslide Processes with Force Histories from Seismological Inversion and Terrain Features. Geophysical Journal International. Retrieved from https://doi.org/10.1093/gji/ggaa269 (ggaa269) doi: $10.1093 /$ gji/ggaa269 